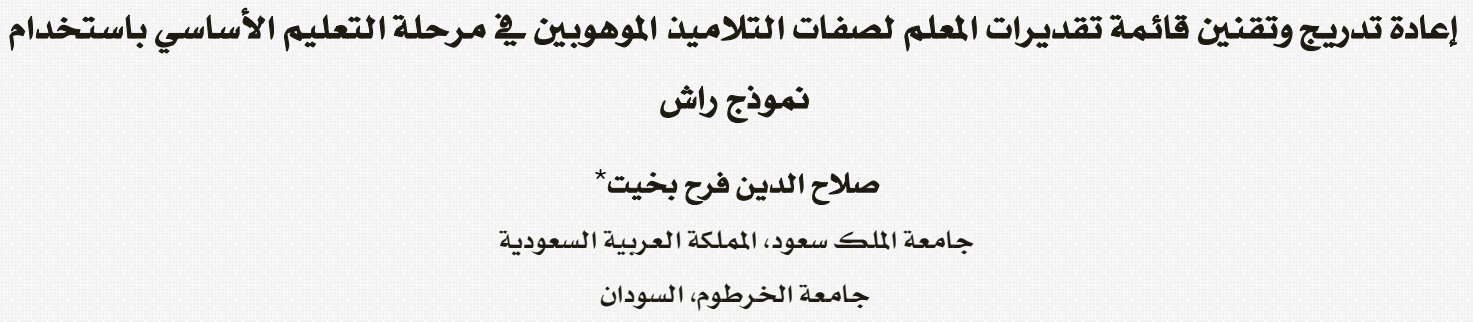

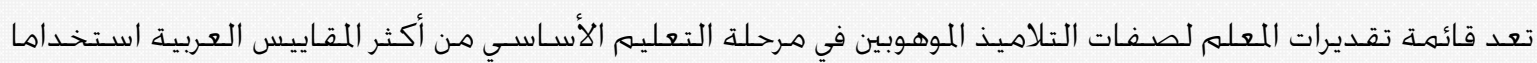

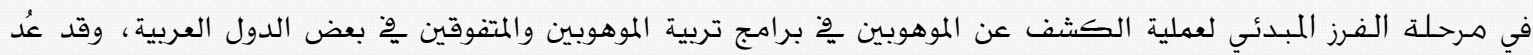
اعتمادها على النظرية الكلاسيكية يخّا القياس أحد عيوبها الأساسية. هدفت هذه الدراسة إلى إعادة تدريج قائمة تقديرات المعلم

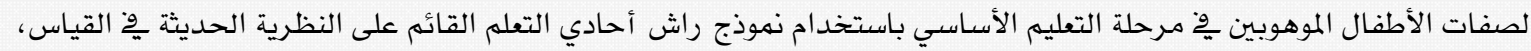
وإلى التوصل لمعايير مختلفة تفسر من خلالها مستويات السمة للأفراد. تم تطبيق القائمة على تلاميذ مرحلة الأساس بالعاصمة العادئ

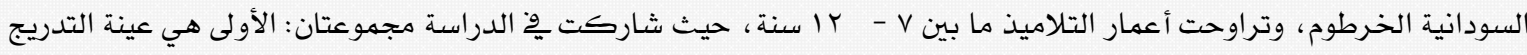

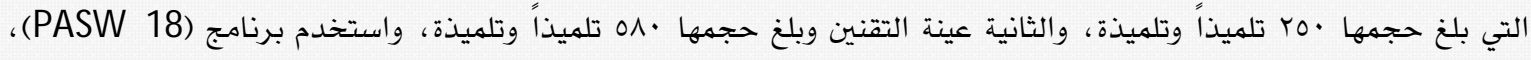

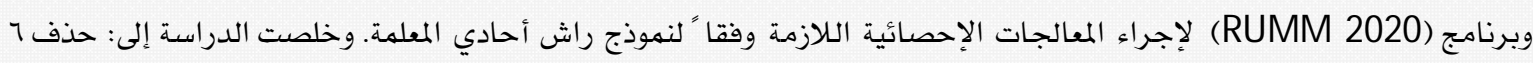

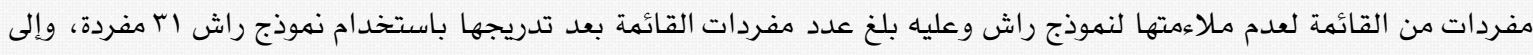
اشتقاق معايير جديدة للقائمة عن طريق إيجاد الدرجات التائية المناظرة للتقديرات المختلفة لقدرة الأفراد. الكلمات المفتاحيه: الموهوبون، النظرية الحـديثة في القياس، نموذج راش، تقدير المعلم للموهوب.

\title{
Re-calibrating and Re-standardization of Teacher Rating Scale of the Characteristics of G ifted Children in Basic Education Phase Using Rasch M odel
}

\author{
Salah F. Bakhiet* \\ King Saud University, Kingdom of Saudi Arabia \\ khartoom University, Sudan
}

The A rabic scale for teachers' rating of basic education gifted students' characteristics is considered one of the commonest A rabic measures used for initial identification of gifted students in some A rabic countries. One of the shortcomings of this scale, however, is that it is based on the classical theory of measurement. This study sought to recal ibrate and renormal ize the scale in the light of Rasch's unidemisional model which rests upon the modern theory of measurement and to develop different criteria for interpreting the levels of individuals' traits. The scale was administered to 830 Basic Education students in Khartoum (age ranged from 7 to 12 years). Two groups of students participated in the study: a calibration sample $(\mathrm{N}=250$ ) and a standardization sample $(\mathrm{N}=580$ ). The statistical treatments were performed using the PSAW 18 and RUMM 2020 programs according to Rasch's unidimentional model. Six of the scale items were deleted for not conforming to Rasch's model. This left the scale with 31 items. Besides, new criteria for the scale were developed by obtaining the tscores that match the various ratings of the individuals' ability.

Keyw ords: gifted students, modern theory of measurement, Rash model, teacher rating of gifted students. *slh9999@yahoo.com 
وبينت دراسـات الكسـو (991) المستخلصـة هـن تطبيق

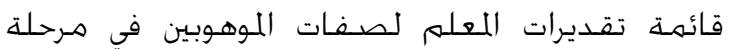

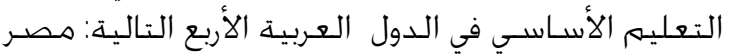

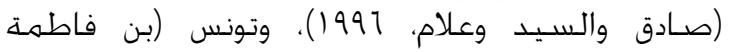

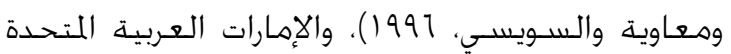

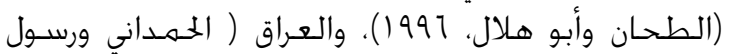

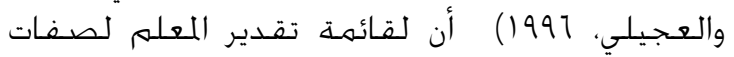
التلاميذ الموهوبين المميزات التالية:

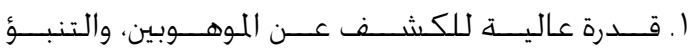

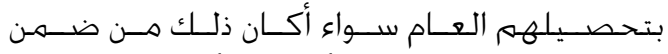

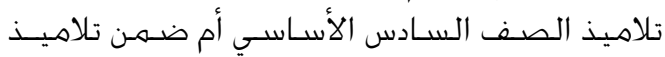

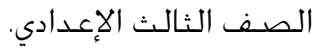

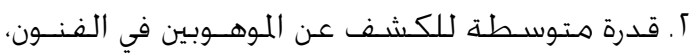

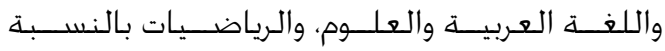

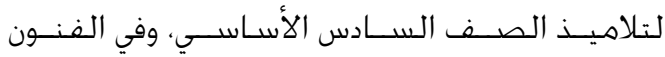

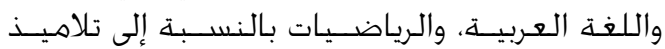

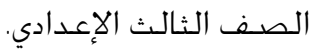

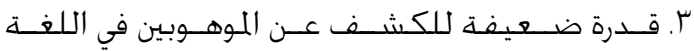
العربية من ضـهـن تلاميذ الصـف الثالث الإعدادي.

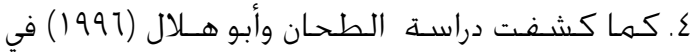

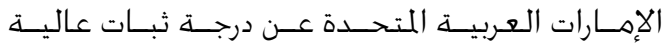

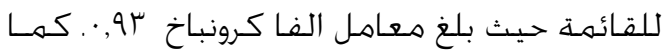

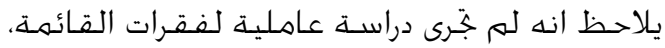

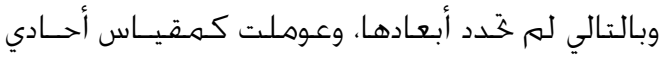

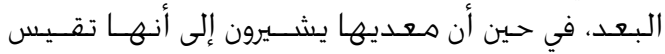

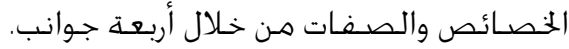

وقد قام عطا الله (1 ؟) بتطبيق قائمة تقديرات المعلم

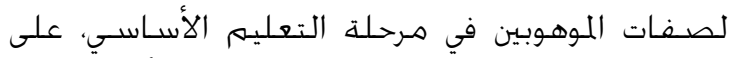

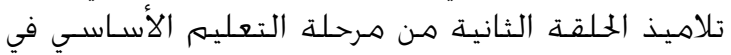

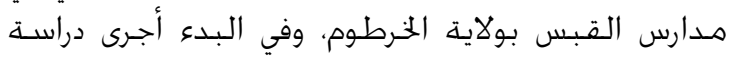

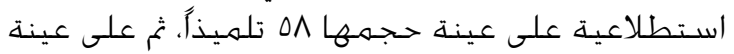

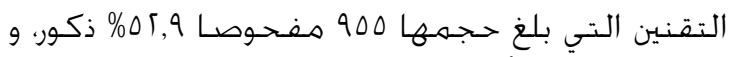

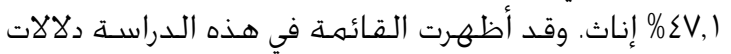

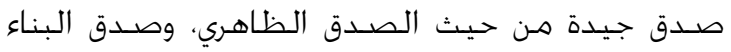

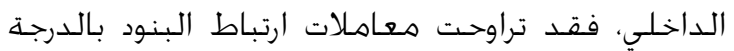

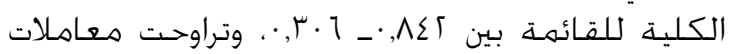

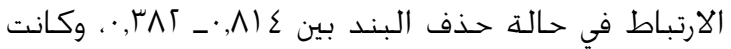

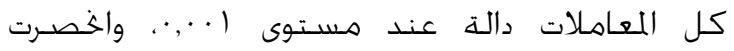

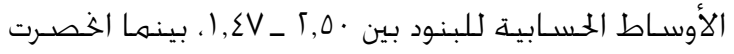

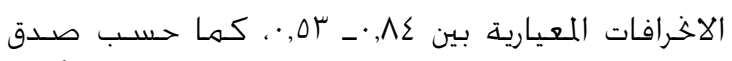

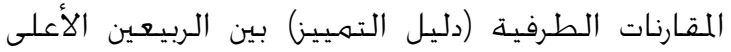

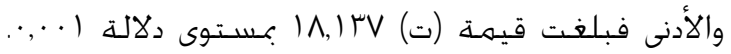

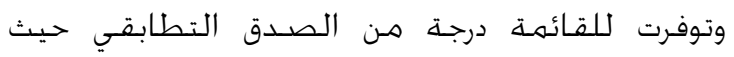

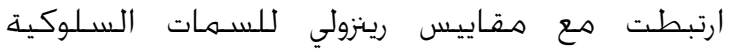
(SRBCSS)

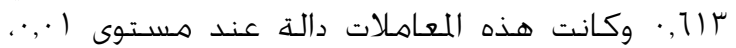

صـدرت قائمة تقديرات المعلم لصـفـات التلاميذ الموهوبين

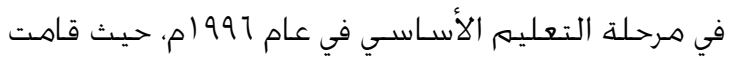

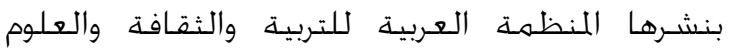

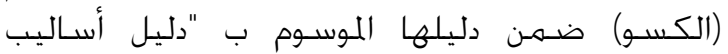

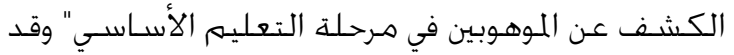

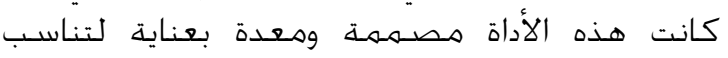

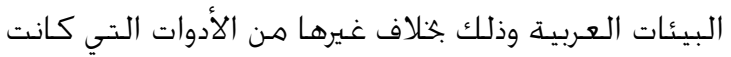

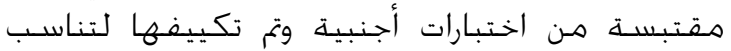

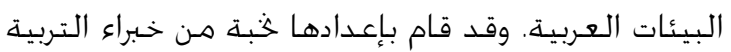

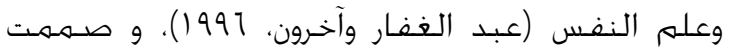

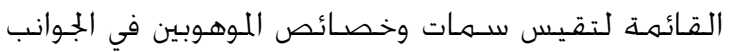

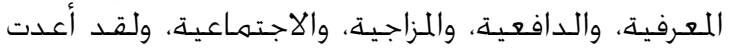

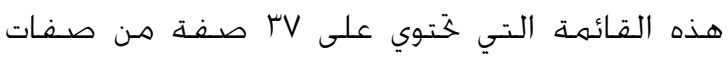

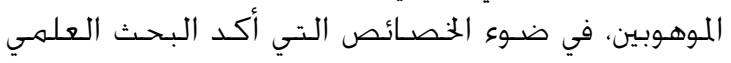

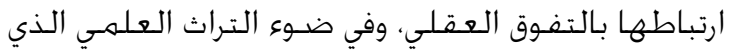

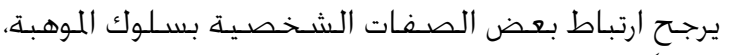

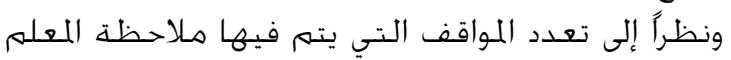

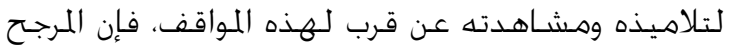

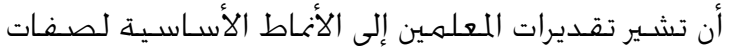

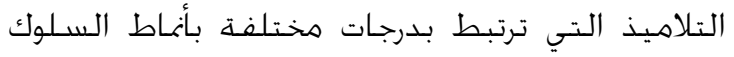

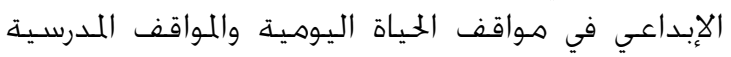

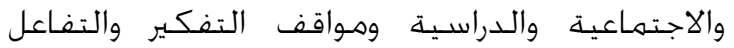

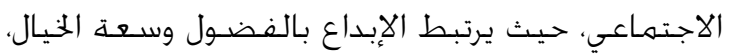

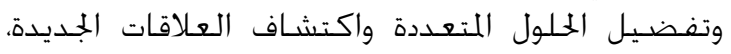

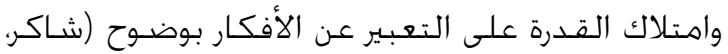
(1)990

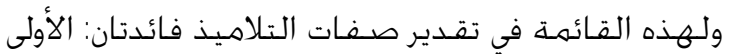

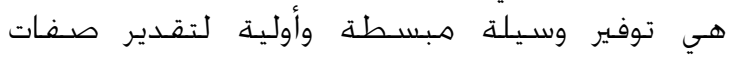

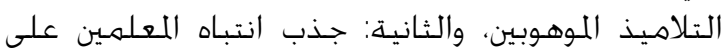

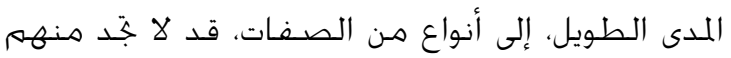
الاهتهمام الكافي في الأحسوال العادية، أضفـف إلى ذلك ألك أنها

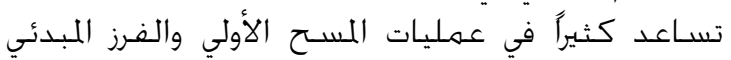

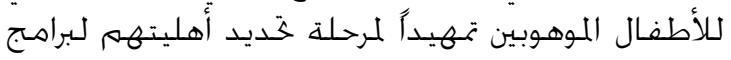
الموهبة والتفوق.

وتعد قائمة تقـديرات المعلم لصـفـات التلاميذ الموهوبين

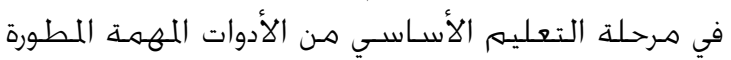

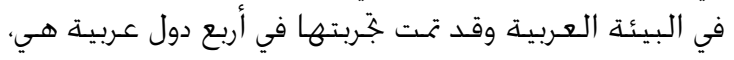

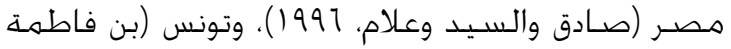

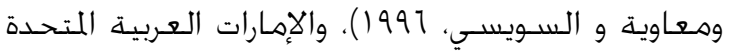

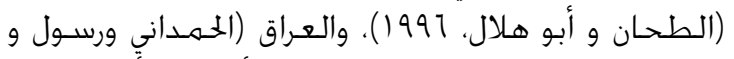

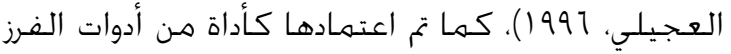

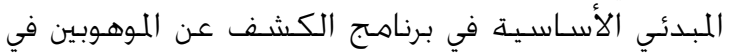

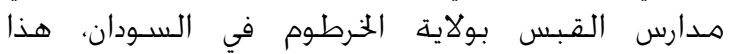

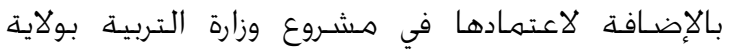

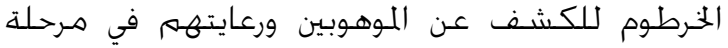
التـرشـيـحات. 
ولأهـمية هذه القـوائم في تقـدير خصائص وسـهـات

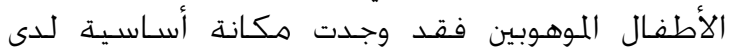

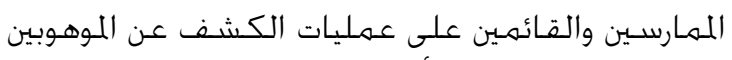

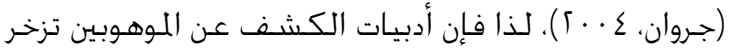

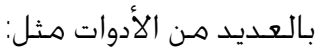

"Scales for rating the behavioral قائمة Characteristics of superior students" تقدير الخصائص السـلوكية للأطفـال الموهوبين مةابن

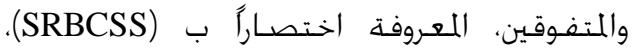

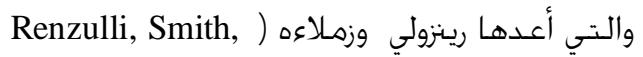
White, Callahan, and Hrtman,1976)، والتي ومهن

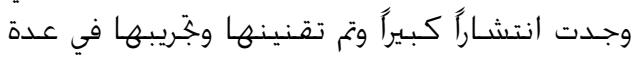
دول مثل (Elliott, Argulewicz, \& Turco,) ، 1986 ,Argulewicz, 1981; Argulewicz, 1985 وكذلك في عدة دول عربية مثل: السـودية،

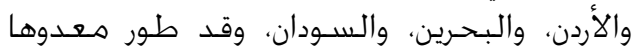
هـورة جديدة لكها (Renzulli et al., 2002).

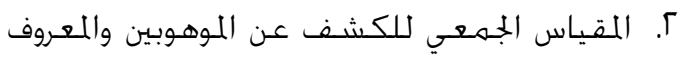
" Group Inventory for Finding (GIFFI) باسئ مـن إعـداد (Rimm,1976 ) وهو هـ (Rimerests" مقاييس التقرير الذاتي، وقد تم تقنينه في الأردن.

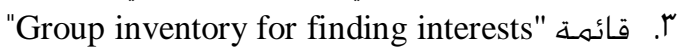

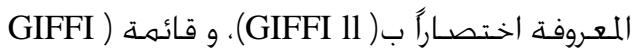

1) هـن إعـداد (Davis \& Rimm, 1974; 1980).

Cummings Checklist of قائهة Characteristics of Gifted and Talented" المعروفة Children" .(Cummings, 1980)(CC)

ه. قائمـ Preschool and Kindergarten Interest

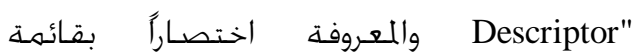
وقد أعدتهها (PRIDE)

7. قائهـة"Eby gifted behavior Index" مـن إعـداد

(Eby, 1983)

Vا. Vائمة "The Purdue academic rating scales Feldhusen, Hoover, \&) التي أعدهـا .(Sayler, 1987

A. Achievement identification measure - قائمة " teacher observation" (Rimm,1988) هـن إعـداد (AIM-TO)

9. The Silverman- Waters Checklist for identifying gifted elementary .(Waters, 1989).school"

"A teacher-based checklist for قائمة identifying giftedness in learning-disabled (Friedrichs, 1990) (students" (من إعـداد.

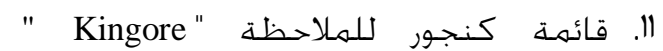
Observation Inventory
بينما كانت نسبة التشـابه \%/V,19 وحسبب الصـدق

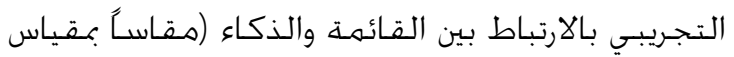

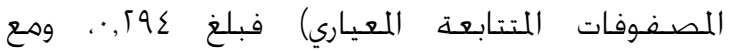

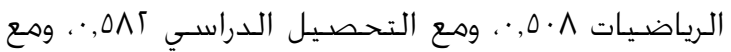

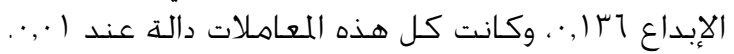

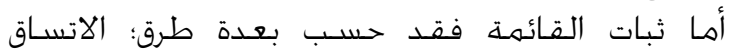

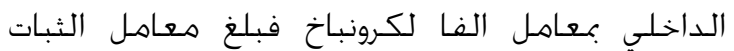
VOA V乏^

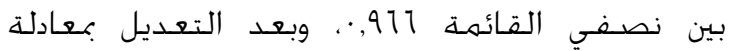

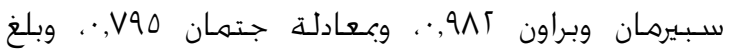

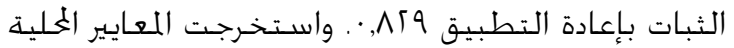

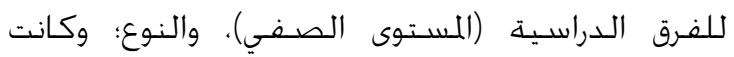
المعايير المستخـدمة هي الدرجات التائية.

وأجريت على القـائمهـ دراسـة أخرى في السـودان حيـث أجهرى

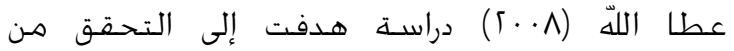

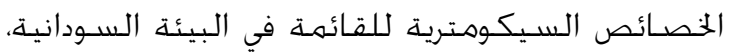

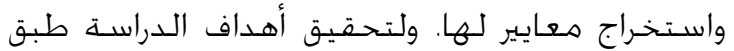

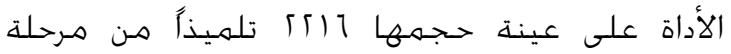

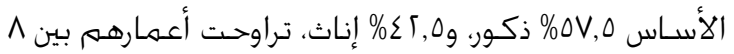

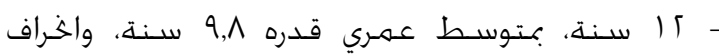
معياري IV,IV. وتم إيجاد صدق لمتوق القائمة بثلاث طرق هي:

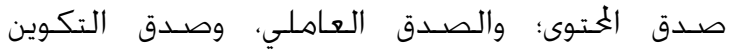

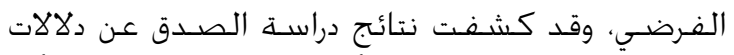

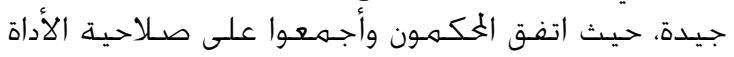

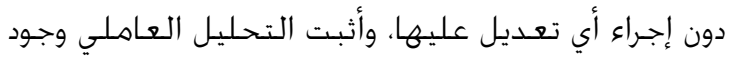

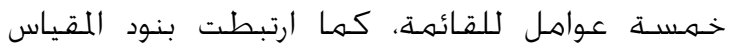

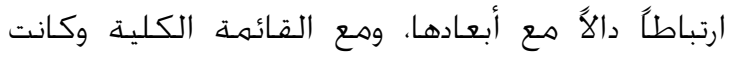

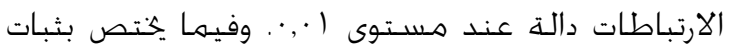

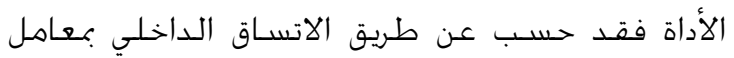

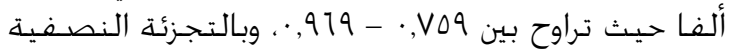

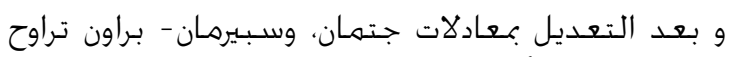

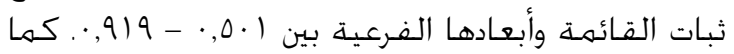

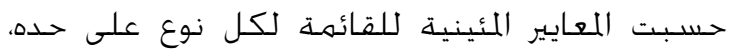

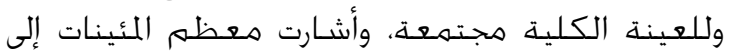
تدرج درجاتها وفقاً للعـمـر الزهـني.

(Elliott, Busse, \& Gresham, 1993; Feldhusen \& ويرى أنه من خـال قيام عدة باحثين بتطوير Heller, 1986)

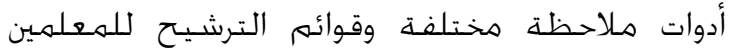

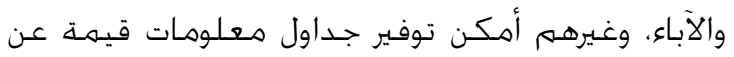

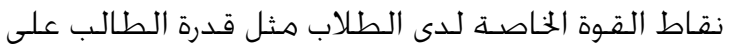

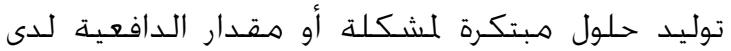

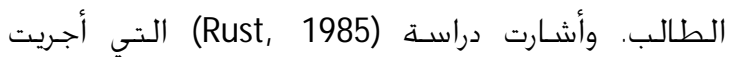

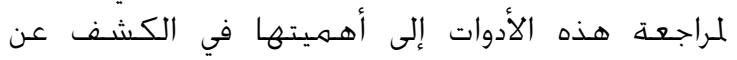
الموهوبين. 
وذلك في دراستـه التي هدفت التعرف على أبرز الخصائص التص

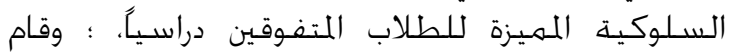

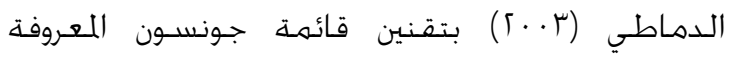

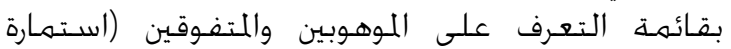

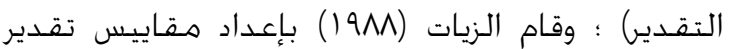

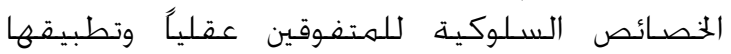

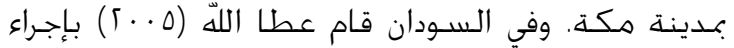

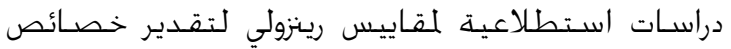

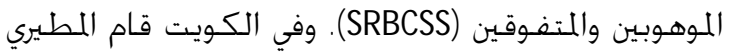

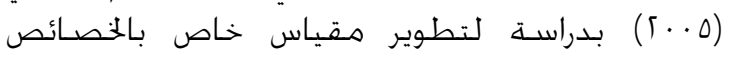
السـلوكية للطلبة الموهوبين في الرياضيات في المرحلة

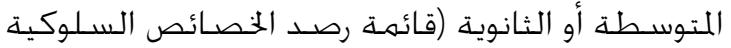

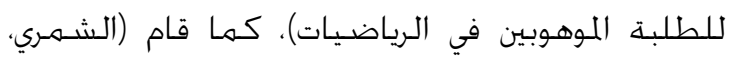

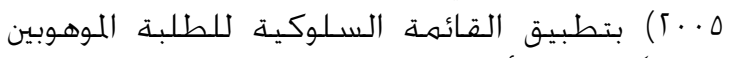

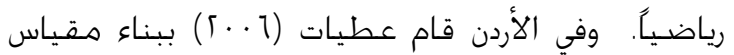

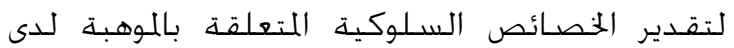

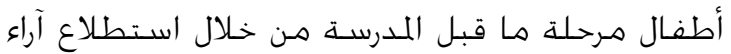

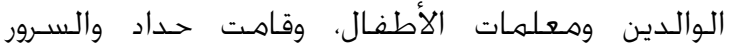

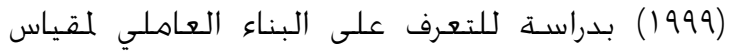

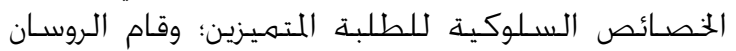

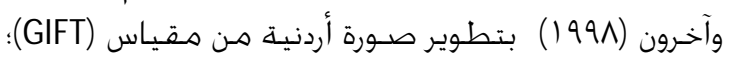

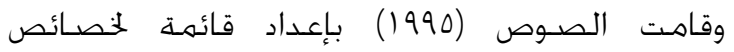

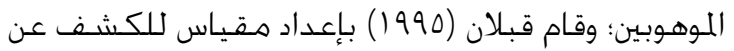

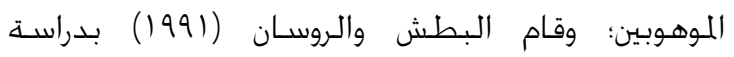

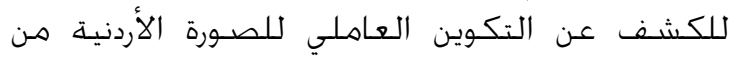

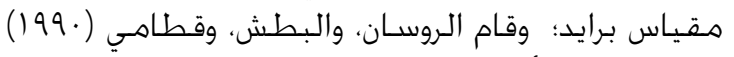

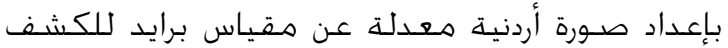

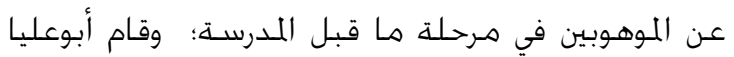

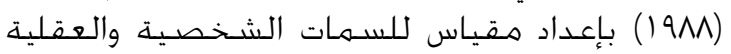
للمبدعين، وقام أيوب (9MM) (19M) بإعداد مقياس للفياس للسـمات

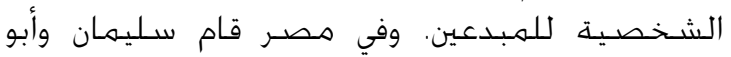

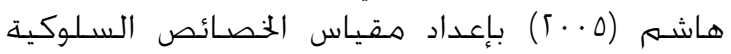

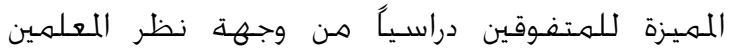

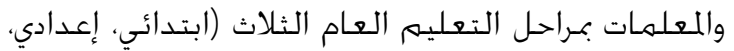

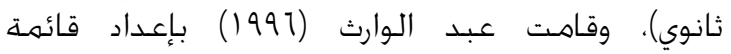

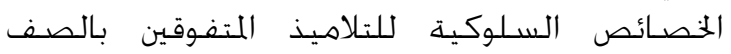
الخامس الابتدائي: وقام منسي (1990) بإعداد قائمة فئه

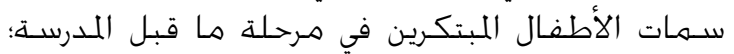

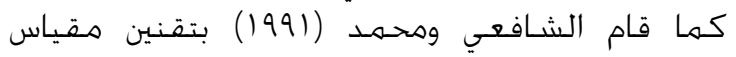
على الأطفال من الخضانة وحتى الصف الخف الخاهس (GIFT)

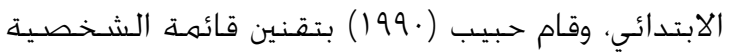

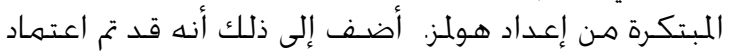

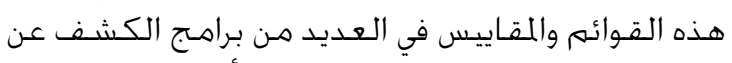
الموهوبين في عدة دول مثل: الكويت، والأردن، والسـودان. لكن يلاحظ أن جميع هذه القوائم والمقاييس والاختبارات

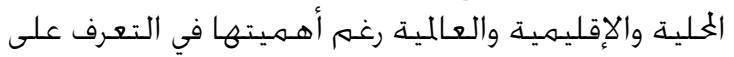

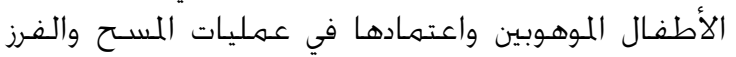

(Vaughn-Neely, 1994) (KOI) المعروفة "Evidence of possible giftedness"

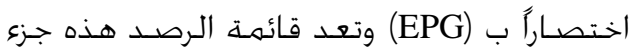

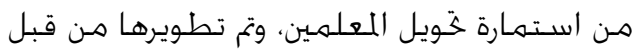

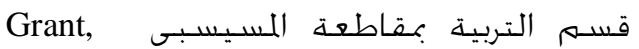

זا. قائهة Hispanic Bilingual Gifted Screening "Instrument" المعروفة اختصـاراً ب (HBGSI) من إعداد (Irby, Lara-Alecio, 1996).

"Gifted \& talented screening form" قائمة (إعة (19) التي أعدها (Johnson,1980).

عا. القائمة التي أعدائها (Silverman,2003) "Characteristics of Giftedness والمسهماة .Scale"

"Scales for Identifying Gifted oاييس" "Students" العـروفة اختصاراً ب(SIGS) من إعداد .(Ryser \& McConnell, 2004) 17. Sقاييس"RENZULLI-HARTMAN SCALES

Scales for Rating the Behavioral) .(Characteristics of Superior Students, 2004 "New Scales for Rating the مقاييس Behavioral Characteristics of Superior Students"( Renzulli, Siegle, Reis, Gavin, \& Reed,2009 ). شا. وقائمة هارتر لـبروفيل التصـور الذاتي (إدراك الذات)

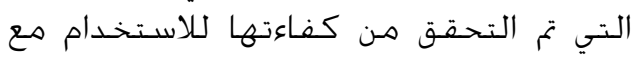
الموهوبين (Rudasill \& Callahan, 2008). \&Pfeiffer( The Gifted Rating Scales 19 مقاييس Jarosewich, 2007; Pfeiffer, Petscher, \& Jarosewich, 2007; Pfeiffer Petscher, 2008; Pfeiffer, Petscher, \& Kumtepe, 2008).

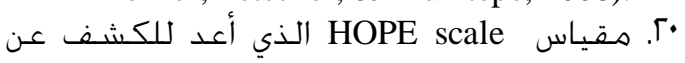

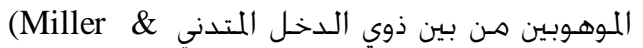
Gentry, 2010; Scott \& Marcia, 2010).

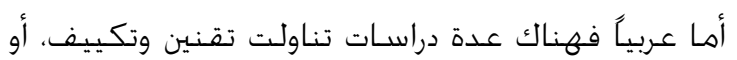

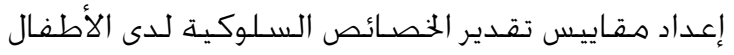

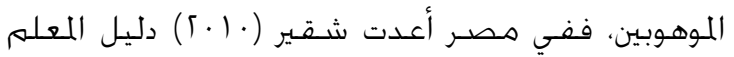

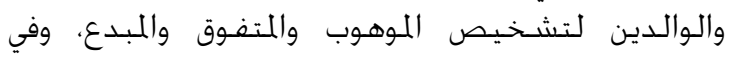

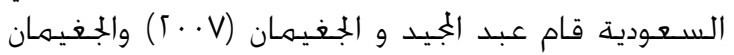

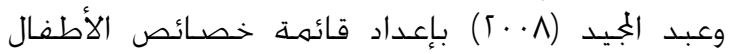

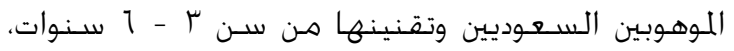

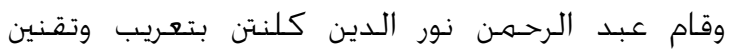

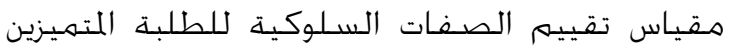

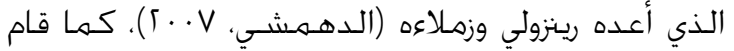

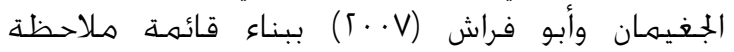

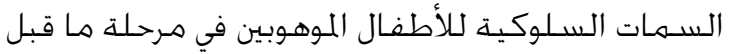

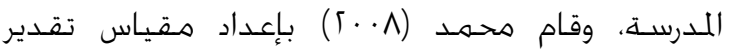
الخصائص السـلوكية للمتفوقين دراسياً بالمرحلة الثانوية المعاسية 


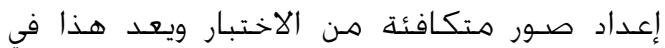

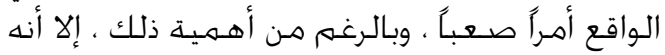

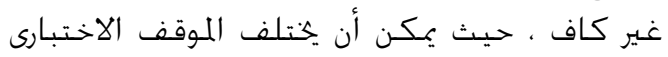
وظروف التطبيق في هاتين المرتين ، الأهـر الذي يؤثر الأنر على دقة ثبات الاختبار

. (Hambleton \& Swaminathan, 1989)

تسـاوي تباين أخطاء القياس لجـميـع أفراد العينة (V)

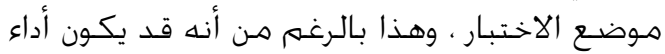

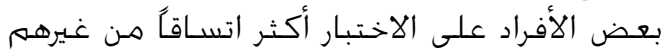

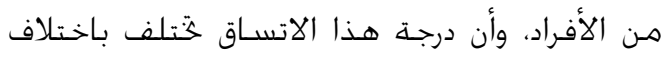

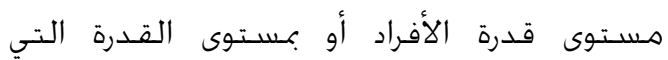

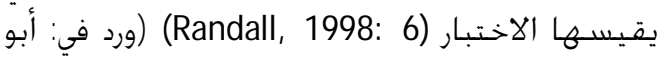

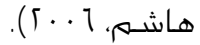

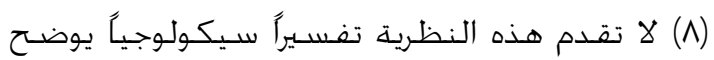

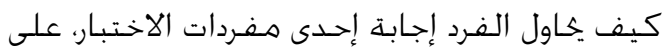

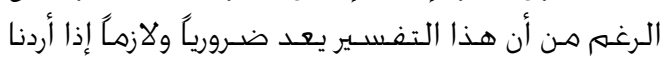

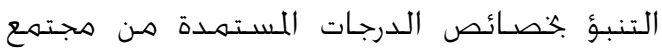
معين أو مجـمعات مختلفـة مـن الأفراد، أو إذا أردنا

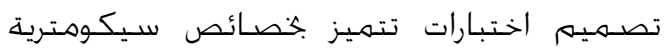

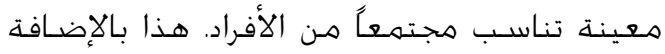

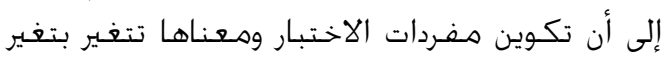

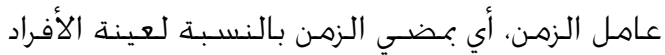

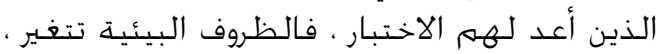

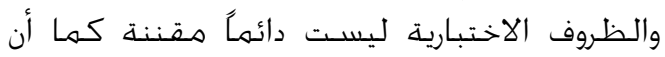

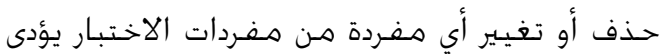

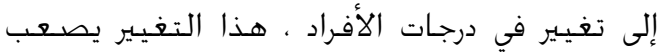

التنبؤ بـه (علام، 1910 ( ).

(9) جـميع خصـائص الاختبارات التي تسـتند في بنائها

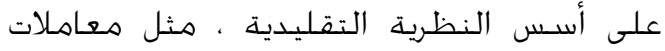

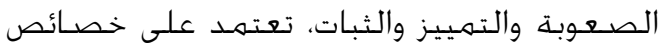

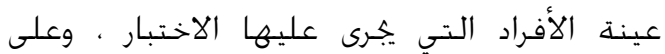

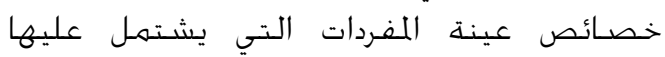
الاختبار (علام، 9AV) ( ) ).

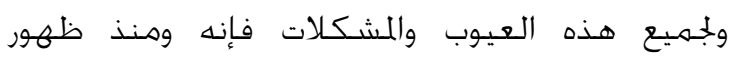

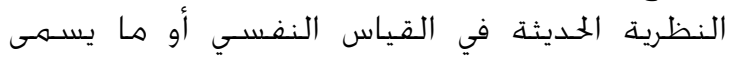

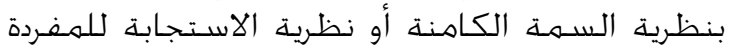

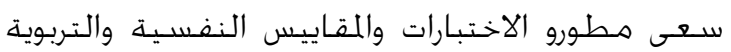

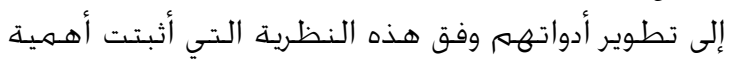

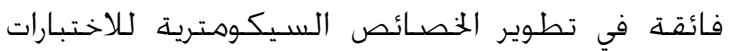
واللقاييس النفسيـة والتربوية.

ولعل المتتبع لحركة البحثث العلمي في ميدان الموهبة

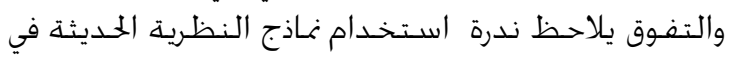

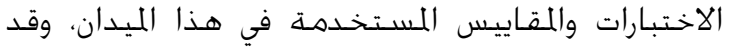

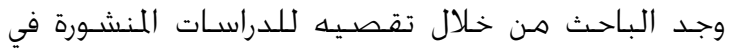

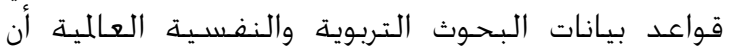

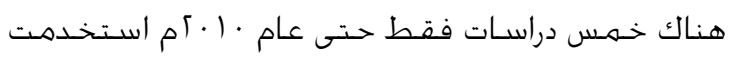

المبدئي للأطفـال الموهوبين، وإسهـامها في معالجـة التحيز

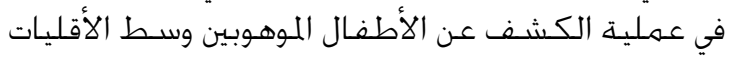
وتقليل تدني نسبة تمثيلهمه في براهـج الموهبة، إلا أنها

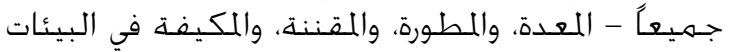

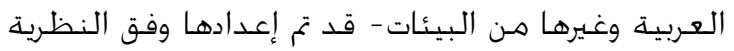

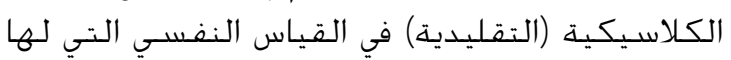

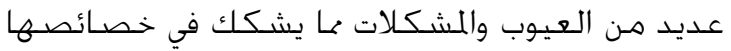

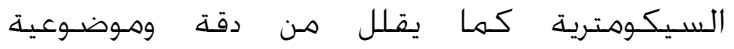
اسـتخـدامها في قياس السـلوك الإنسـاني، وقــ أجهـل

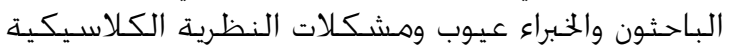

$$
\text { (التقليدية) فيما لئون اخيراع: }
$$

(1) تقيد الدرجـة الكلية للاختبار ببنود الاختبار، انعدام

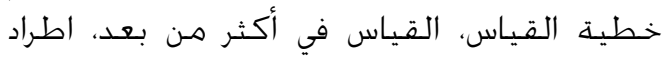

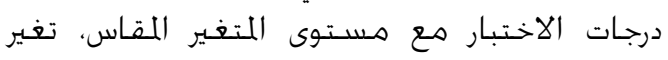

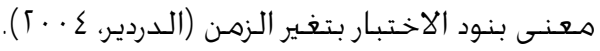

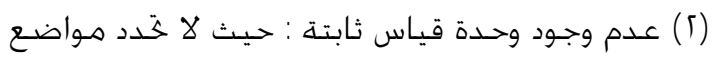

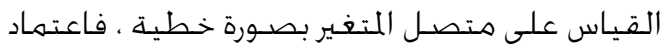

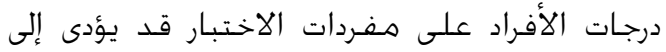

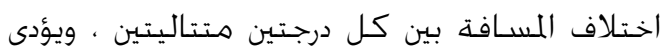

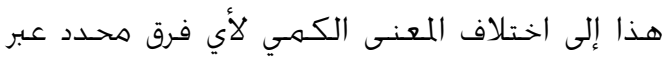

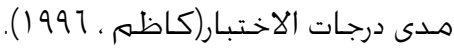

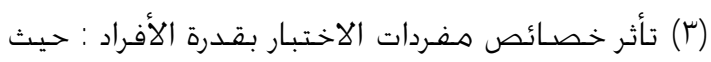

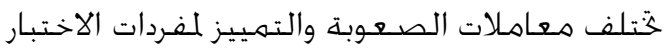

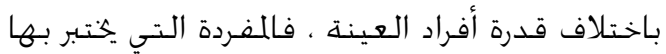

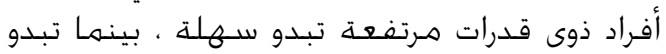

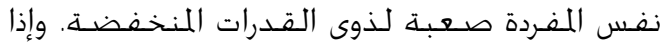

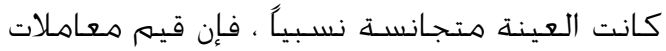

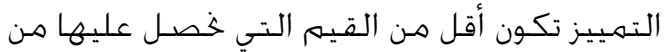

عينـة غير متجانسـة.

.(Hambleton \& Swaminathan, 1989)

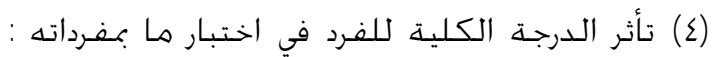

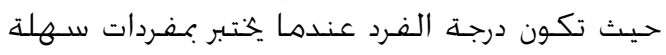

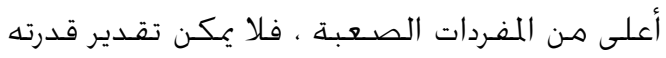

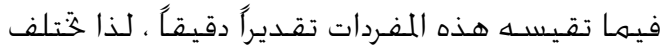

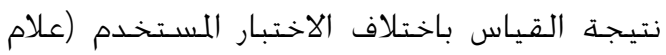

. ( $1 \cdots$. .

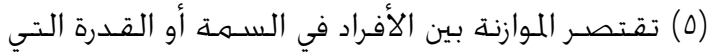

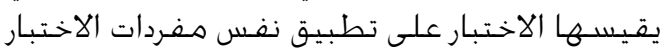

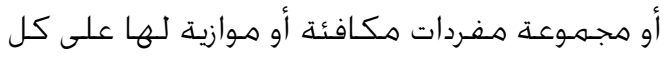

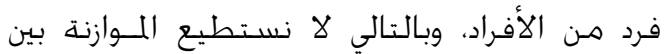

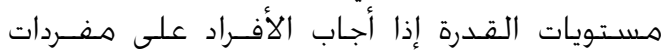

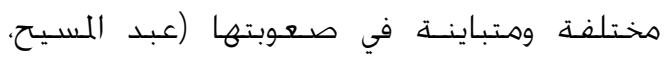

(1) تأثر ثبات الاختبار بالموقف الاختباري : حيـث يعتمد الاخد

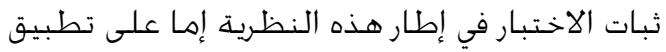

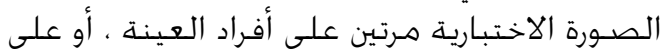




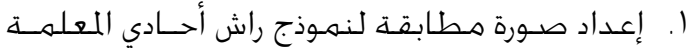

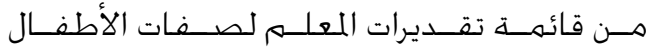
الموهوبين في مرحلة التعليم الأسـاسي، تتوفر فيها لـانها

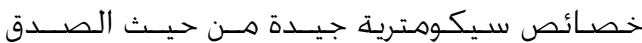

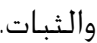

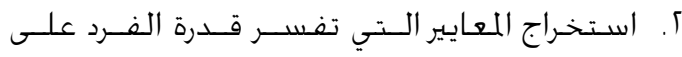

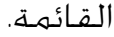

\section{أهمية الدراسة:}

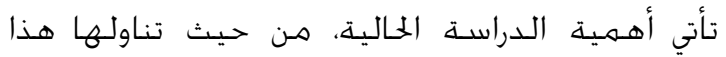
الموضوع الهام في مجال تطوير المقاييس والاختبارات

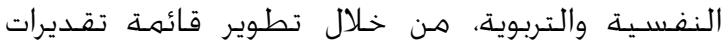

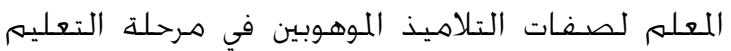

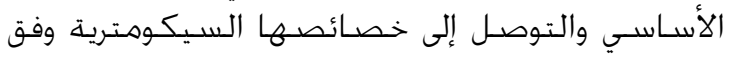

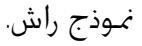
كما تكمين أهمية الدراسـة الحالية في أنها تزود ميدان

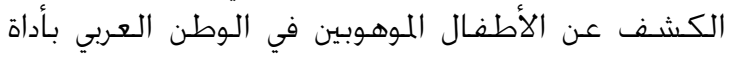

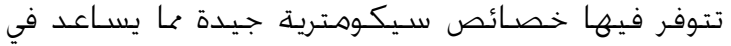
تقليل الأخطاء الشائعة في هذا الجمال، كما تعد نتائج

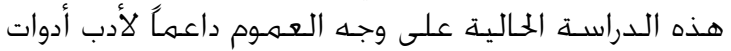

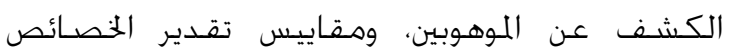

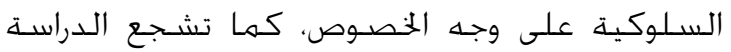

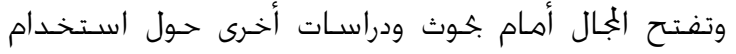

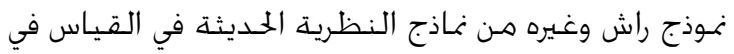

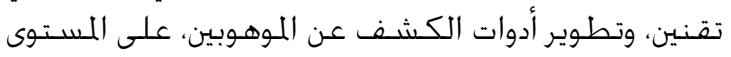
العلـي والإقليهـي.

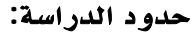

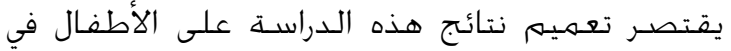

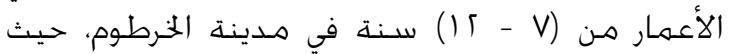

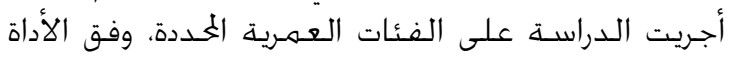

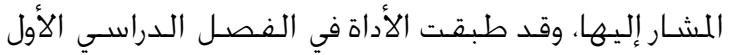

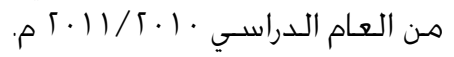

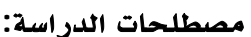

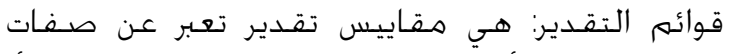

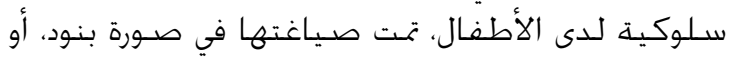

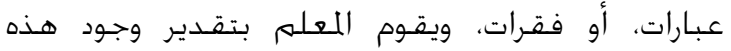

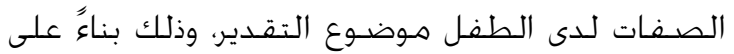

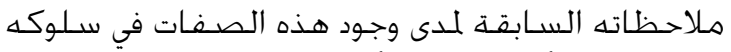
بدرجة كبيرة، أو متوسطة، أو ضعيفة أودهة

صفات الأطفال الموهوبين: هي مجموعة مختلفة منسة نسبيًً

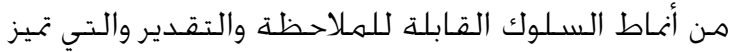

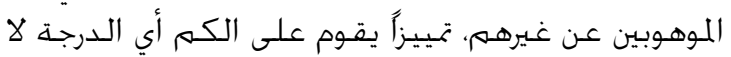

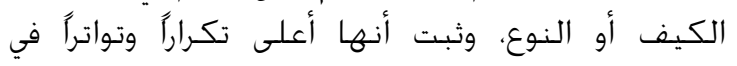

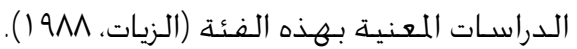

هذه النماذج الحديثة في القياس في اختبارات وهقاييس

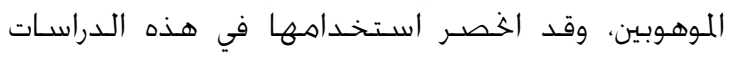

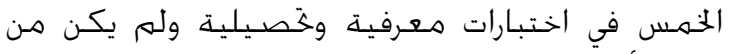
بينها أي استخـدام في قوائم ومقاييس تقدير وفير وقوائم شطب الخصائص والسـمات السـلوكية للموهوبين والمتفوقين.

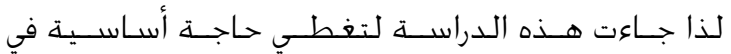

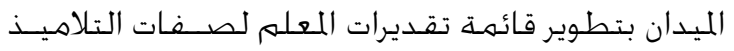

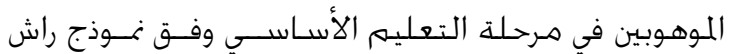

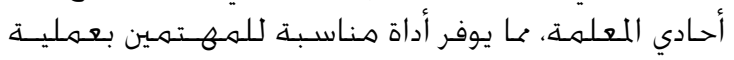

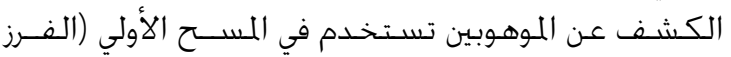
المبدئي).

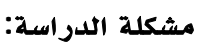
ظل مجال الكشـفــ عـن الأطفــال الموهــوبين في الـوطن

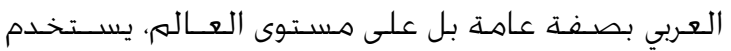

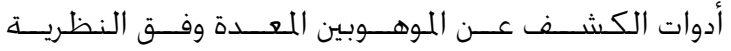

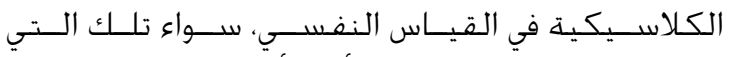

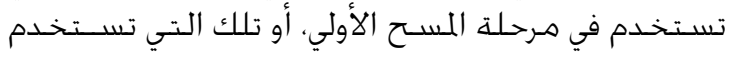

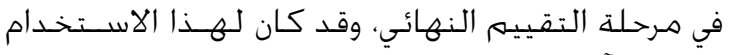

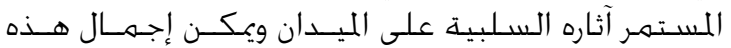

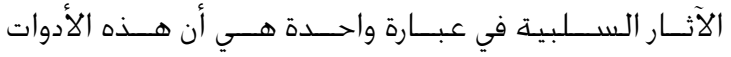

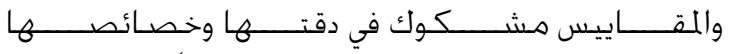

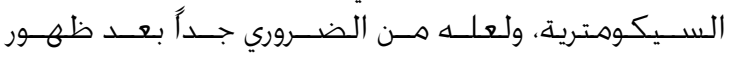

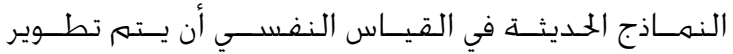

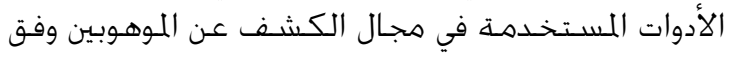

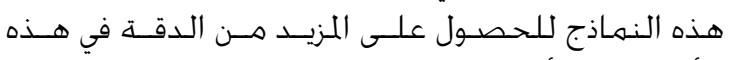

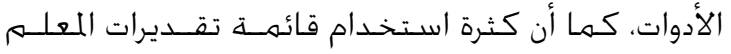

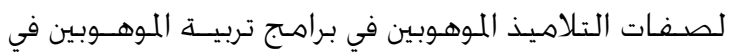

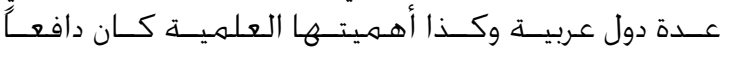

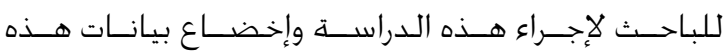
القائمة لنمـوذج راش محاولًا الإجابة على الأسئلة الآتية: ا. ما مدى تطابق مفردات قائمة تقديرات المعلم لصفات التلاميذ الموهوبين في مرحلة ائلة التعليه

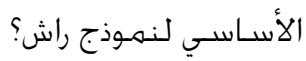

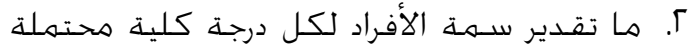

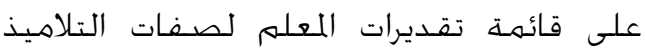

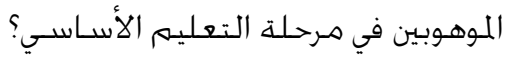

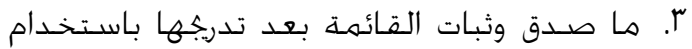

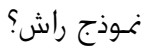
ع. ما معايير القائمة بعد تدريجها باستخدام نموذج راش

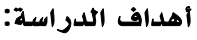
تســـى الــراســة إلى خحقيق الأهـداف التـالية: 
إرجاع الاختلاف بين الأفراد إلي خصـائص الأفراد وليس إلي

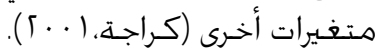

: Classical Theory النظرية التقليدية (الكلاسيكية)

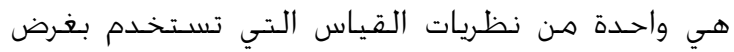

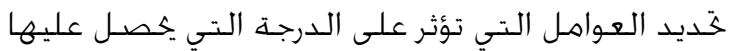

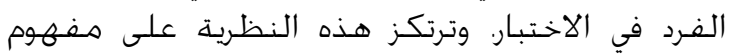

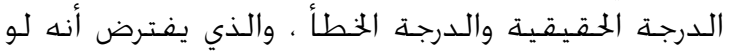

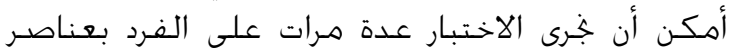

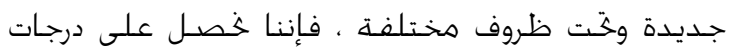

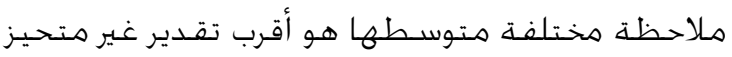

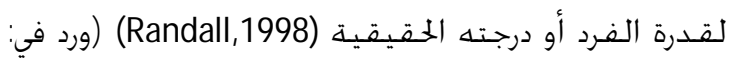

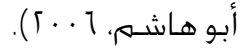

\section{الطريقة والإجراءات}

المشاركون في الدراسة:

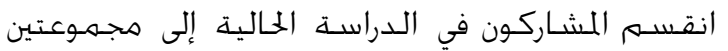

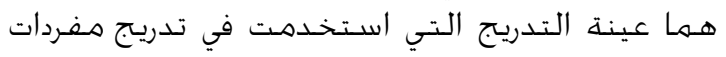

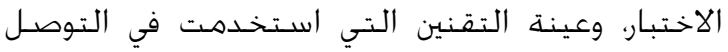
لمعايير القائمة.

أ. عينة التدريج: تم تطبيق الاختبار على عينة

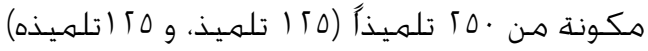

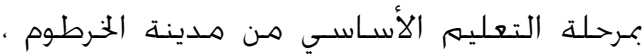

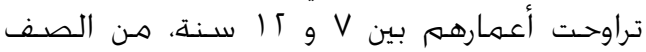

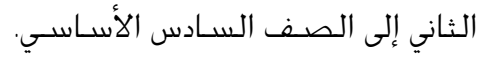

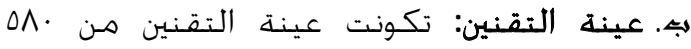

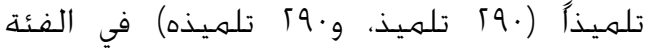

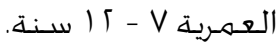

أداة الدراسة: - إس

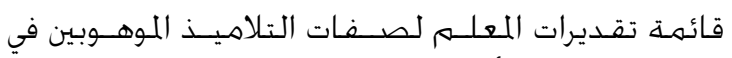

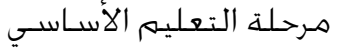

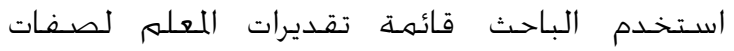

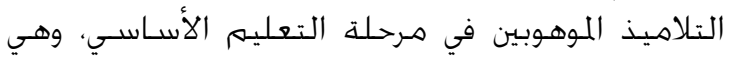

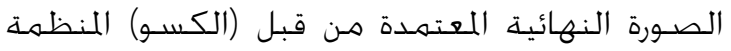

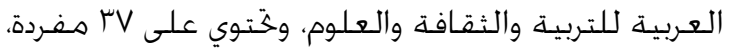

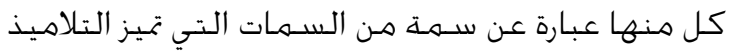
الموهوبين، تتناول الجوانب المعرفية، والدافعية، والمزاجية، والاجتماعية للموهوب.

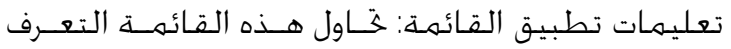

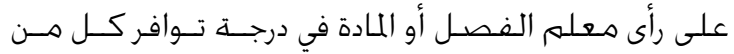

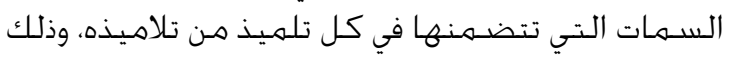

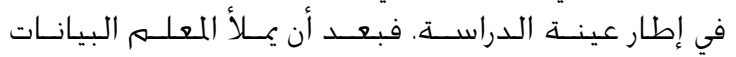

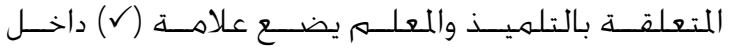

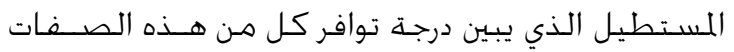

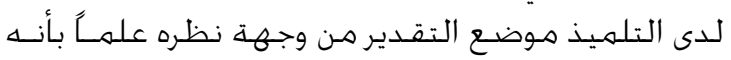

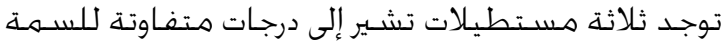

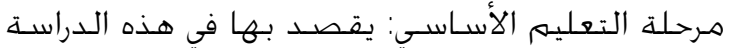

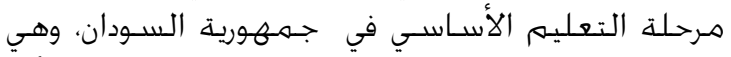

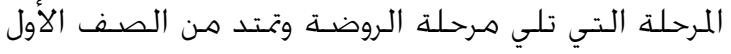

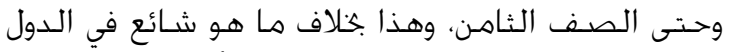

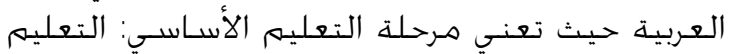

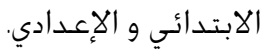

نموذج راش: يعد نموذج (راش Rasch) أهم نماذج السـمات

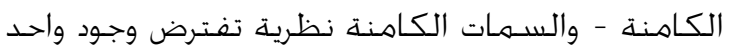

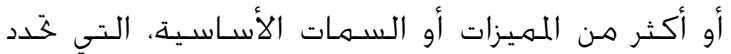

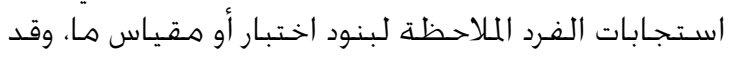

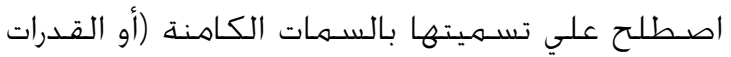

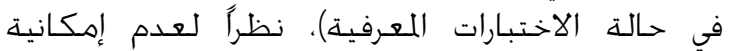

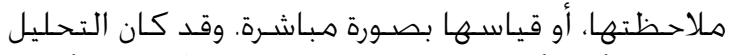

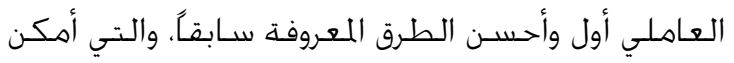

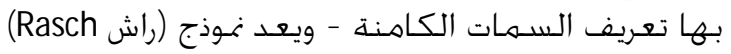

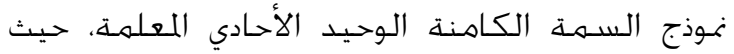

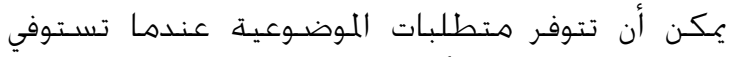
فروض النموذج وهي:أحادية البعد. واستقليالية القياس.

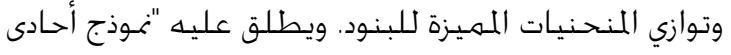

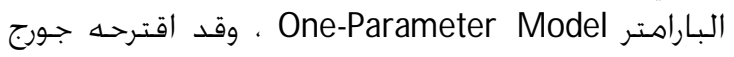

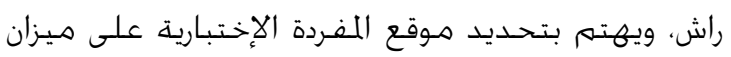

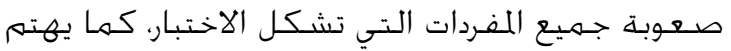

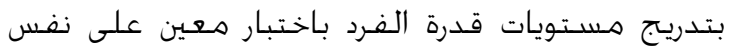
ميزان تعبير المفردات (علام ، ... ؟).

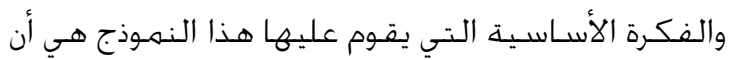

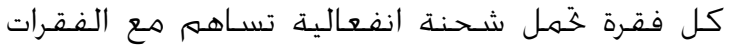

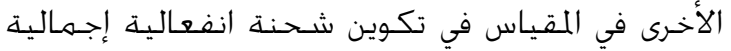

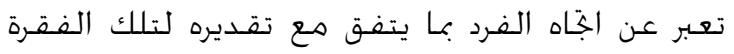

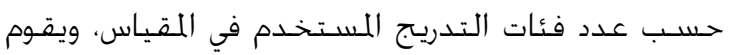

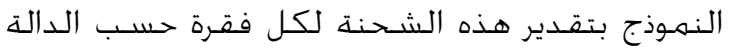

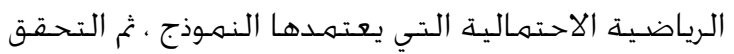

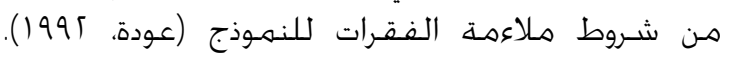

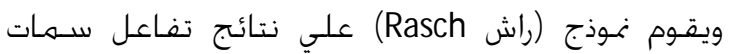

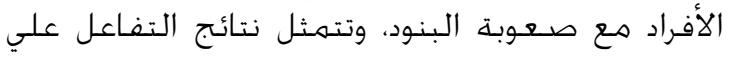

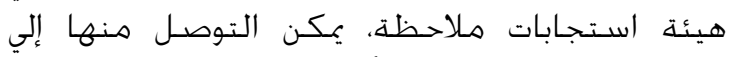

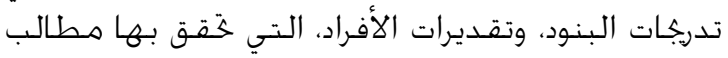

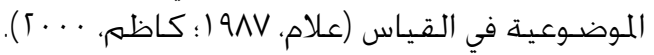

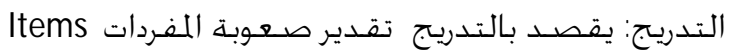

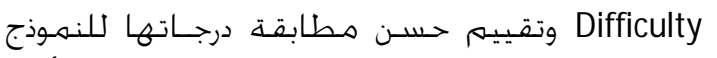

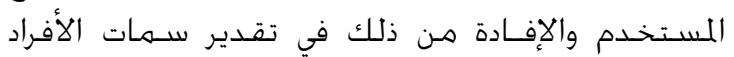

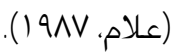

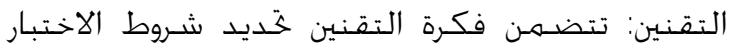

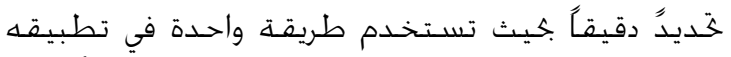

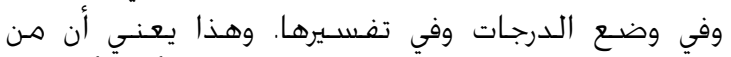

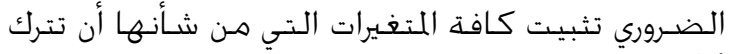

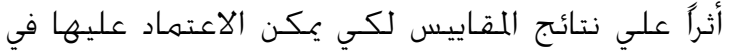


قدرته عندئذ أدنى من المدى الذي يغطيه المقياس.

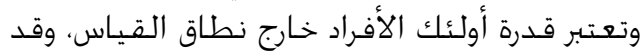

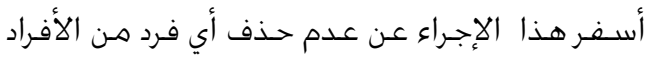

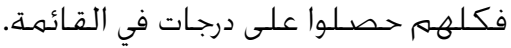

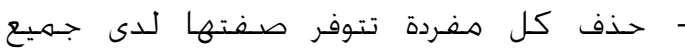

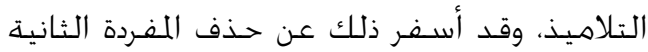
من مفردات القائمة، وبذلك تبقت في في القائمة

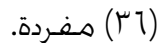
- حذف كل مفردة لا تتوفر صفتها لدى أي مـن

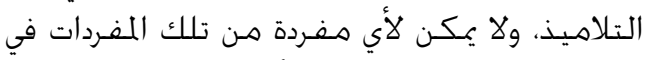

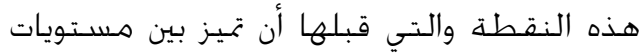
المتغير، وله يته حذف أي مفردة وفقاً لـهذا الإجراء.

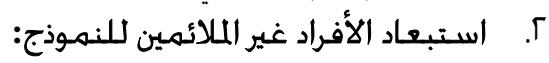

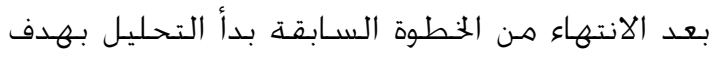

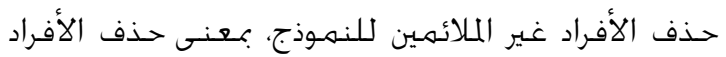

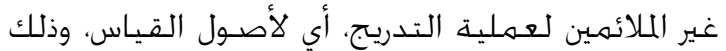

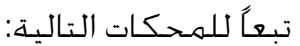

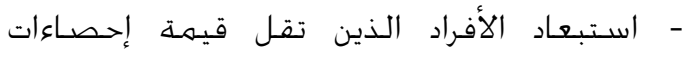

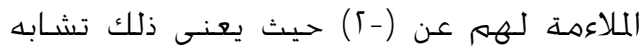

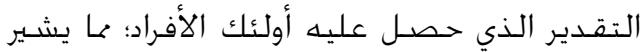
إلى عدم صـدق الاستجابات.

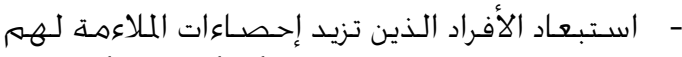

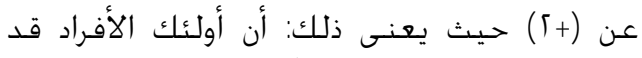

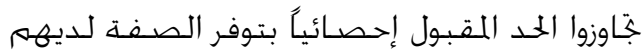
عن المفردات التي تزيد في مستوى توفرها عن إلتون

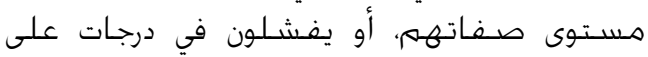
مفردات تتطلب صفات أقل من صفاتهاتهم: ميات

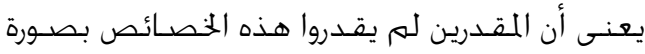
دقيقة لديهم: ألمهرين.

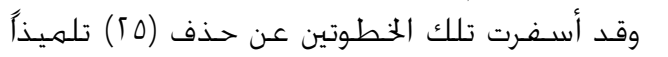

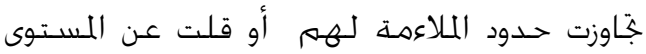

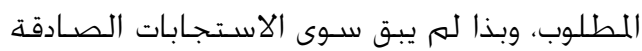

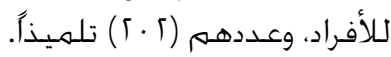

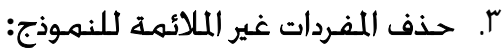

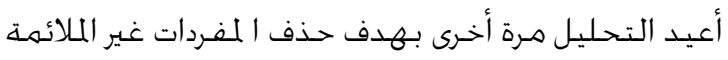

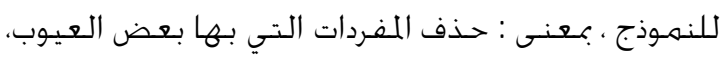

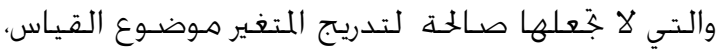
وذلك تبعا للمحكات التالية:

- حذف ا لمفردات الني تقل قيم إحصـاءات الملائمة

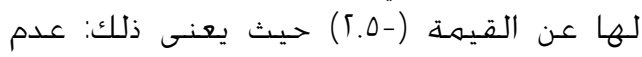
اسـتقلالية تلك المفردات عن باقي مفردات المقياس. الفيا.

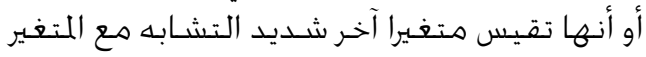
المفترض قياســه.

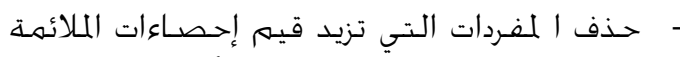

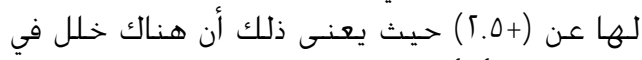
بناء المفردة، أو أنها تقيس متغيرا آخر.

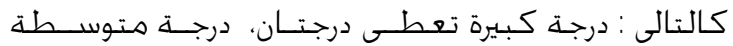

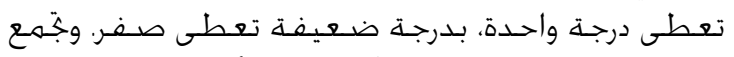

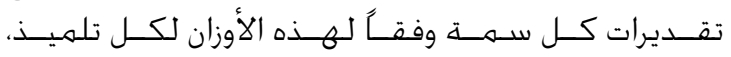

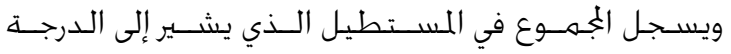
الكلية للتلميذ.

إجراءات الدراسة:

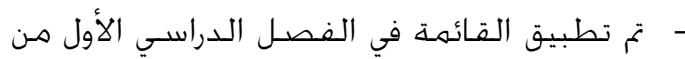

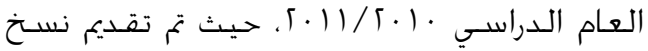

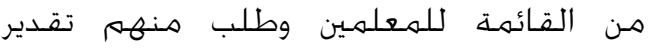
تلاميذهم وفق الصفات الواردة في القائمة، ولا

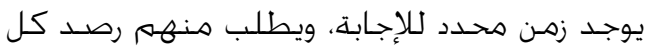

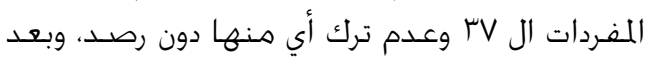

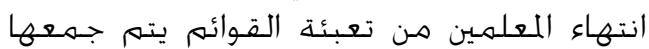

منهم: - تم ترميز الإجابات وإدخالها إلى البرنامج الإحصائي (PASW 18)

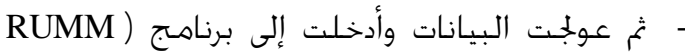

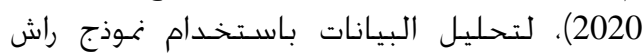
الاحتعالي اللوغاريتهي الأحادي اللعلمة، لتدريتج

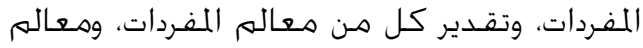

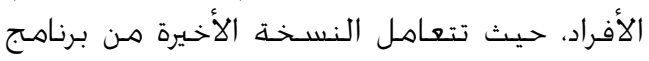
(RUMM 2020)

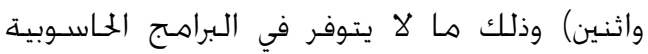

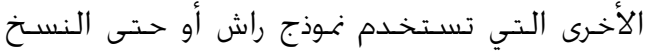

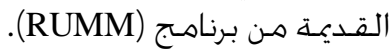

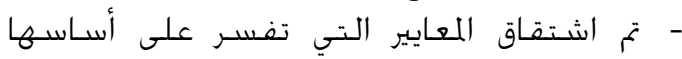
المستويات المختلفة للأفراد.

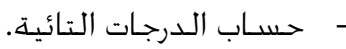

\section{نتائج الدراسة}

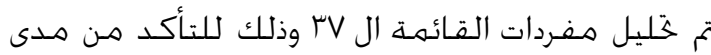

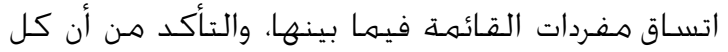

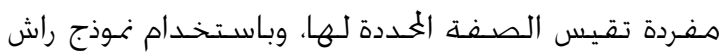

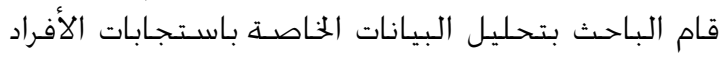

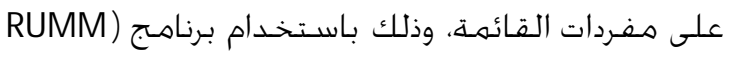

(2020

وفيما يلي نتائج التحليل: ( RUM M 2020$)$

ا. حذف البيانات التامة والصفرية من مصفوفة

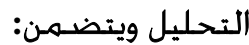

- حذف كل فرد حصل علـ على الدرجة الكاملة في

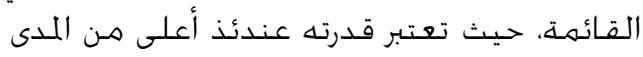

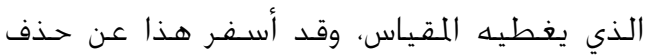

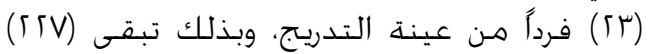
أجري عليهم التحليل.

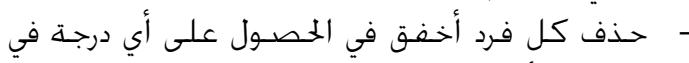

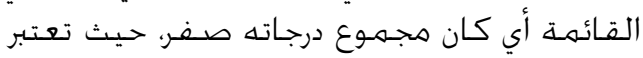




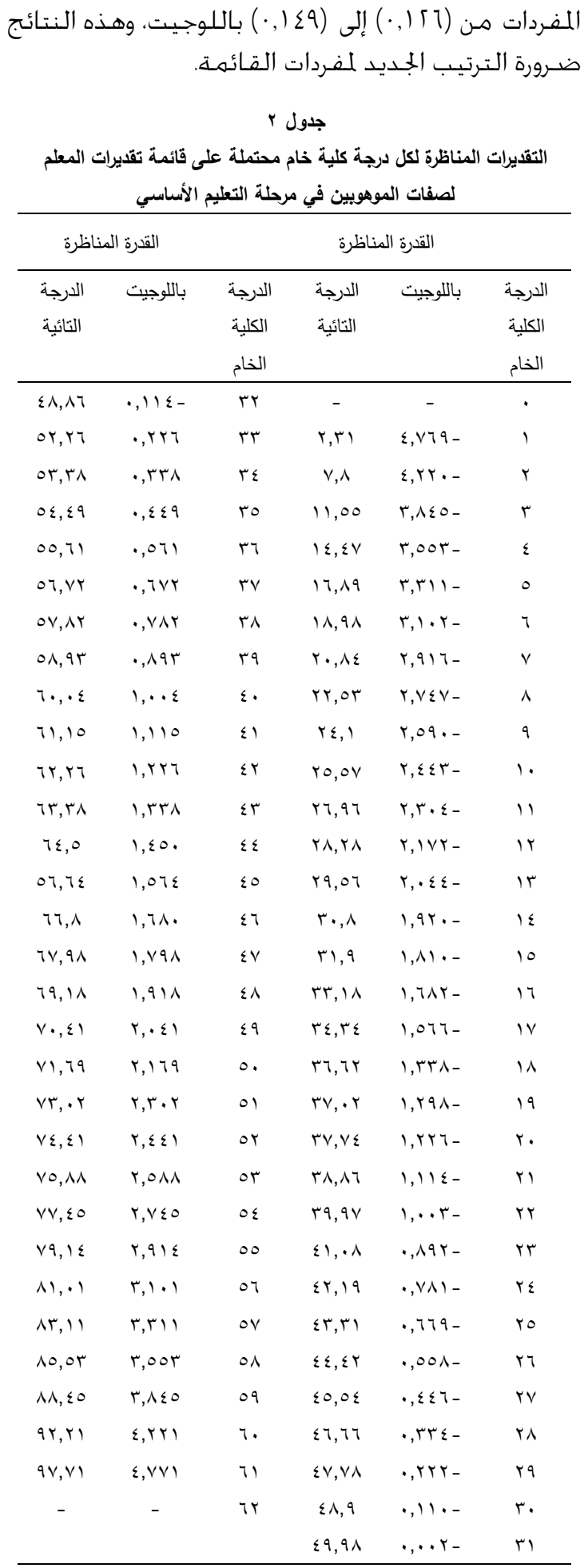

صلدق القائمة:

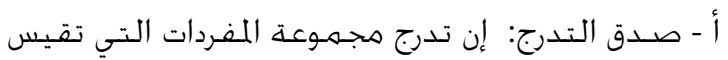

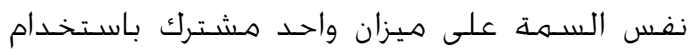

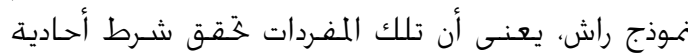

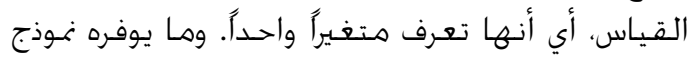

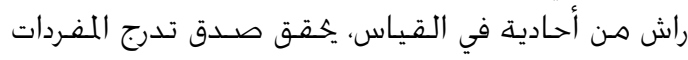

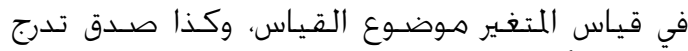

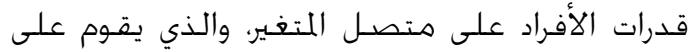

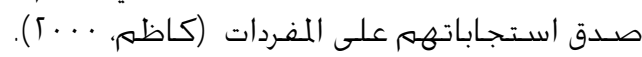

- وقد أسفرت نتائج التحليل عن حذف ه من من

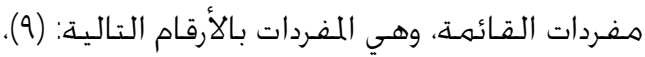

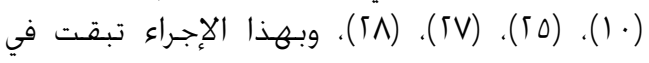

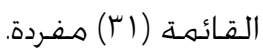

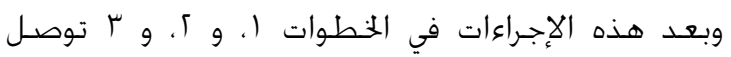

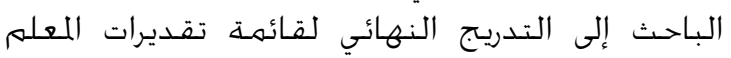

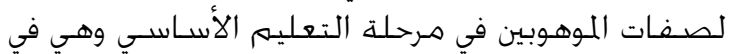

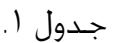

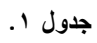

التدريج النهائي لمفردات قائمة تقيرات المعلم لصفات الموهوبين في مرحلة

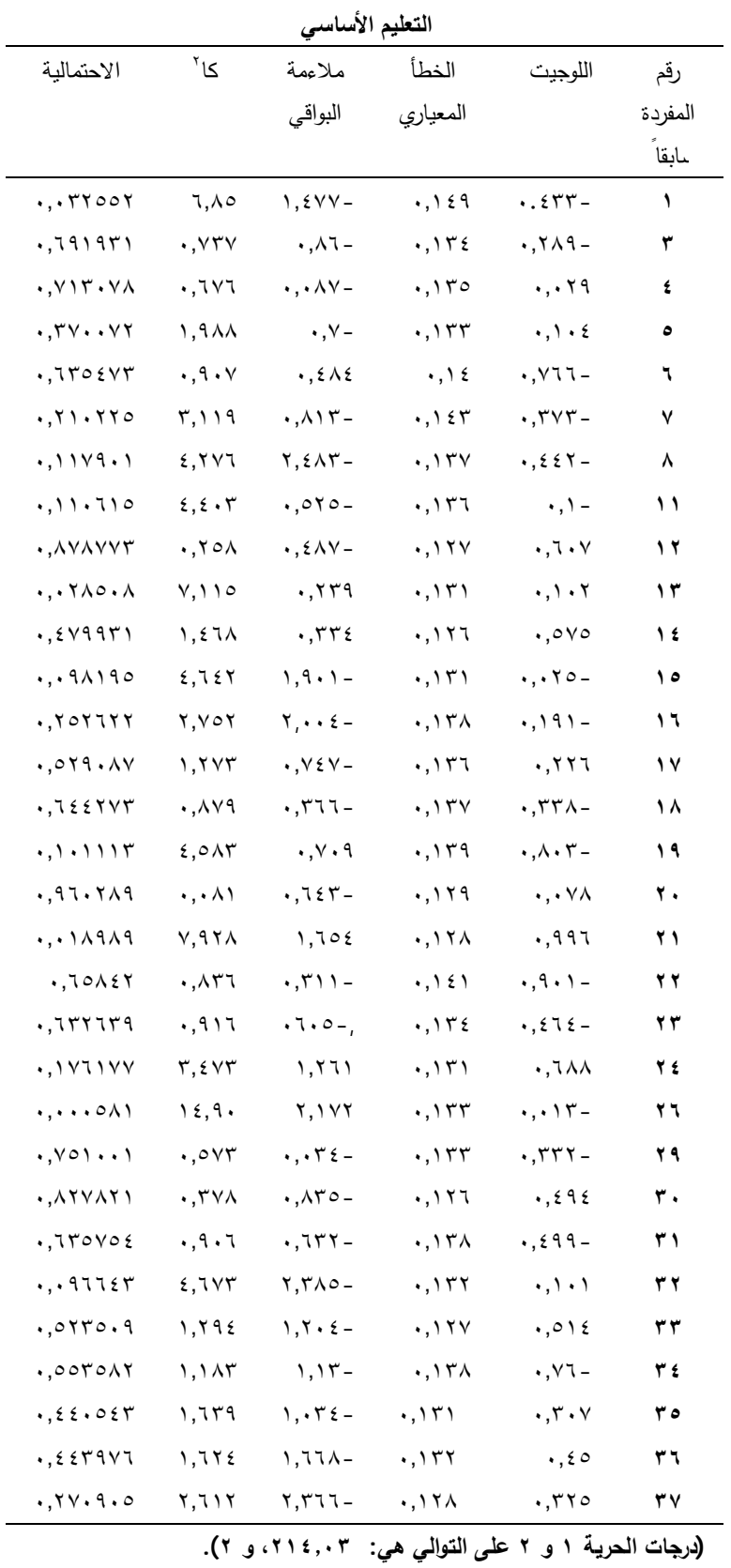

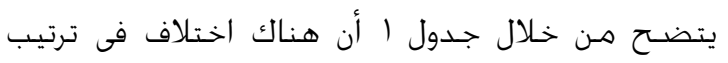

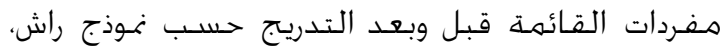

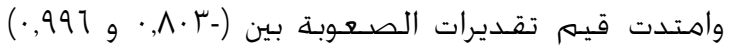

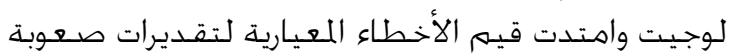


جدول تقـديرات القـدرة المـتملة المقابلة لكل درجـة كلية مككنة على القـائمة.

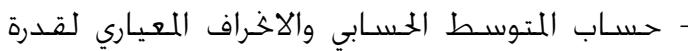

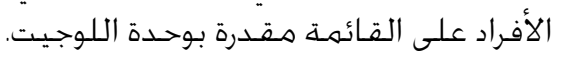

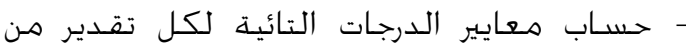
تقـديرات القـدرة المقدرة بوحهدة اللوجيت.

\section{مناقشة النتائج والخلاصة}

هدفت الـراسـة الحالية إلى إعـادة تـدريج قائمة تقديرات

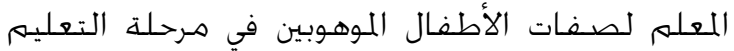

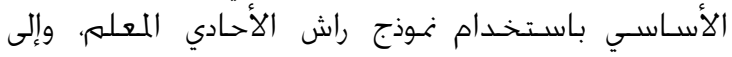

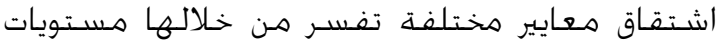

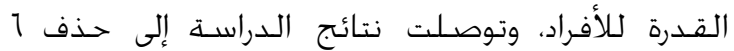

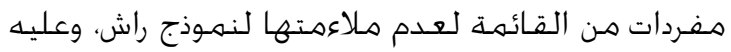

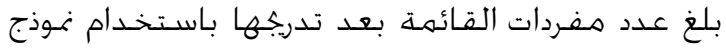

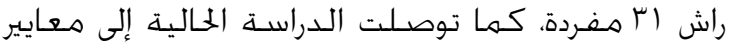

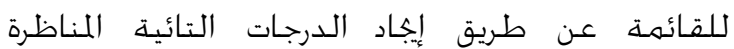

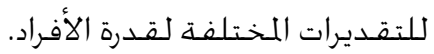

ويتضـح مـن ذلك أنه أمكـن لهذه الدراسـة الاسـفـادة هـن

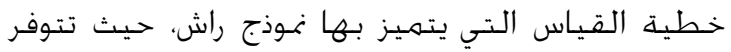

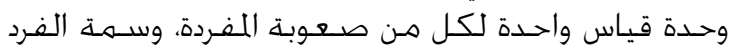

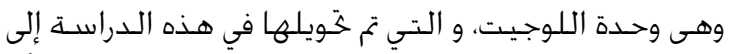

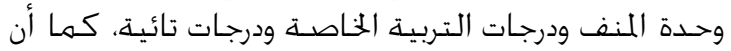

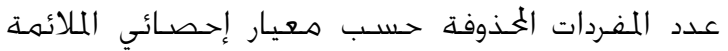

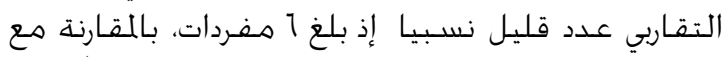

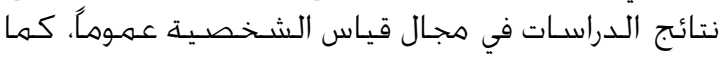

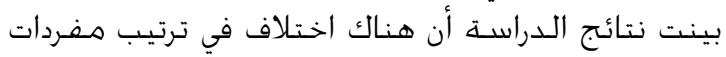

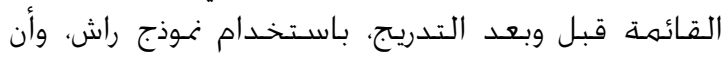

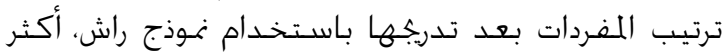

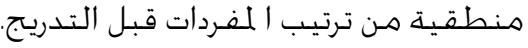

ونتائج هذه الدراسـة تلفت أنظـار المختصـين في براهـج

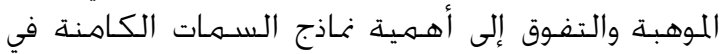

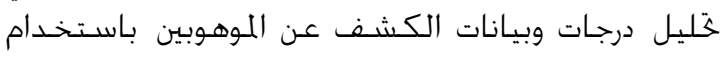

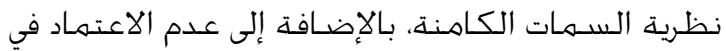

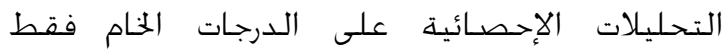

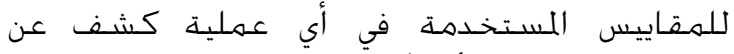

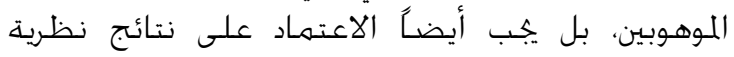

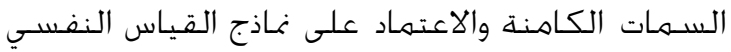

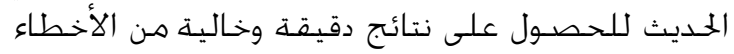

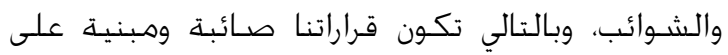

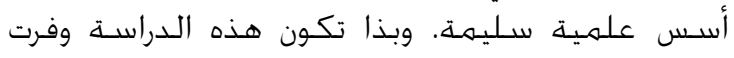

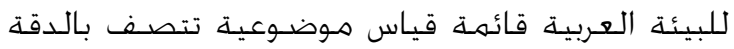

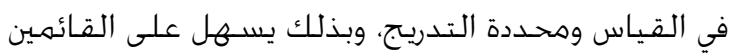

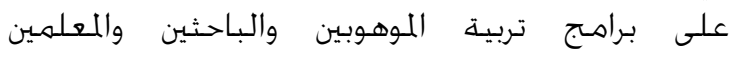

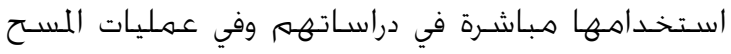

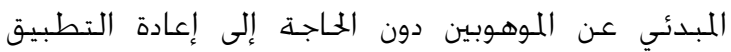

وتتحقق أحادية البعد بتحقق ملاءهة كل من الأفراد

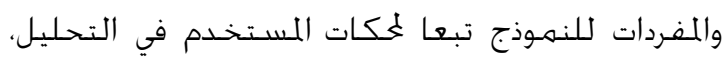

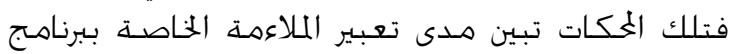
(RUM M 2020 )

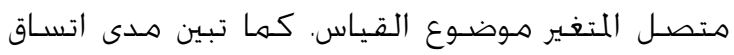

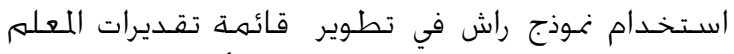
لصفات الموهوبين في مرحسلة التعليم الأسـاسي. فئوني ب - الصـدق العاملي: أجرى الباحث التحليل العاملي فئي

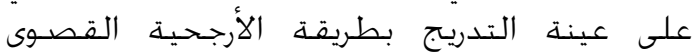

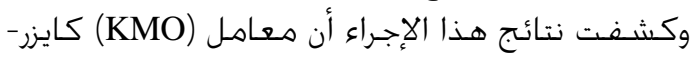

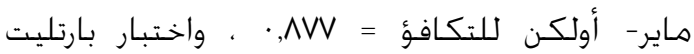

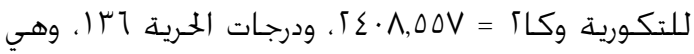

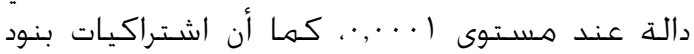

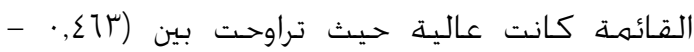

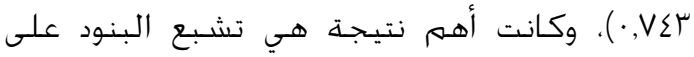

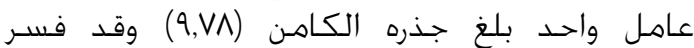

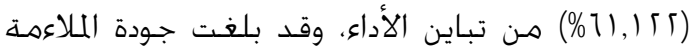

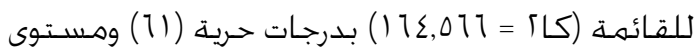

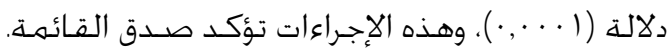

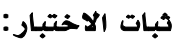

أ -ثبات التدريج: إن تدرج مفردات المقياس على ميزان تدرج

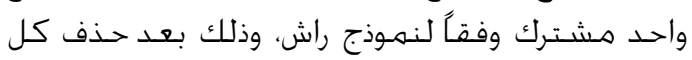

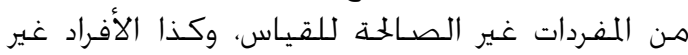

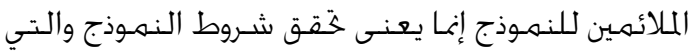

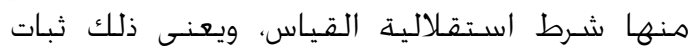
تقديرات كل من الصـوبة الصة والقدرة، وعدم تأثر هـما

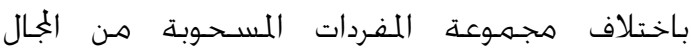
السـلوكي الععين، أو باختلاف عينة الأفراد الذين تم المات تطبيق الاختبار عليهمه.

ب -معامل الثبات الذي يوفره برنامج الحاسب الآلي: يقوم

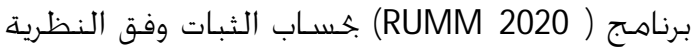
التقليدية للقياس وقد بلغ ثبات القائمة بمعامل الفا

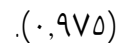

المعايير التي تفسر قدرة الفرد على الاختبار الدرجات

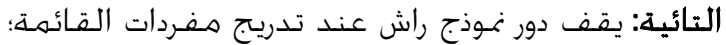

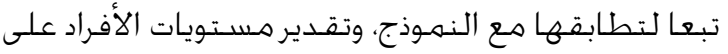

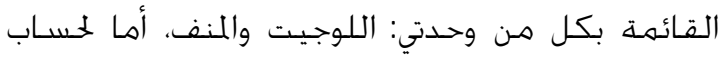

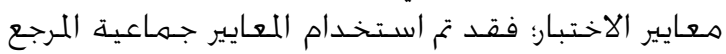

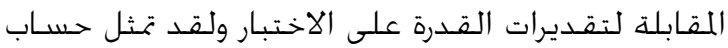
المعايير في القيام بالخطواتلفيرات التالية:

- حسـاب الدرجـة الخام الكلية لكل فرد هـن أفراد عينة

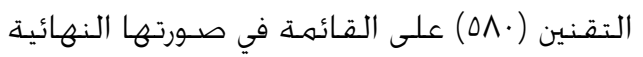

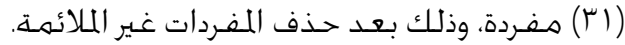

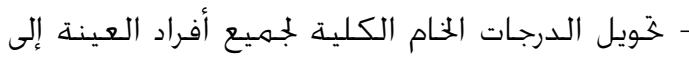
تقـديرات القـدرة المقابلة لها وذلك باستـخدام 
قبل المدرســة دراسـة استطلاعية. الجلة العربية

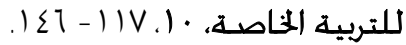

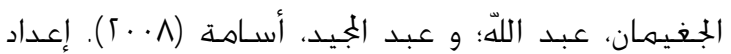
قائمة خطان، خصائص الأطفال الموهوبين السـودوديين

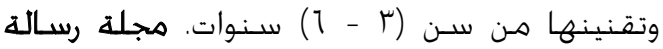

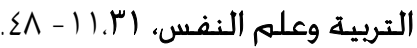

حبيب، مجدي ( • (99) ). اختبار الشخصـية المبتكرة : تأليف

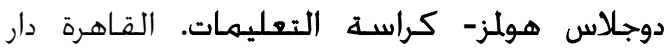
النهــــة المصـرية.

حـداد، عفـاف؛ والسـرور،ناديا (999 (). الخصـائص السـلوكية

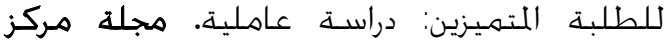

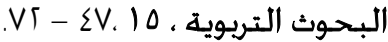

الحـمـداني، مـوفق؛ رسـول، خليل؛ العجيلي، صباح (1997).

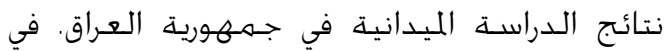

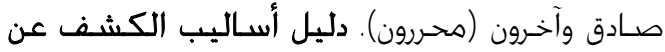

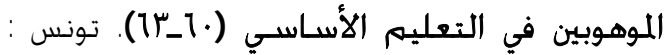

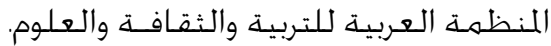

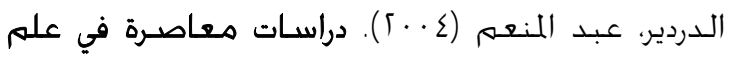

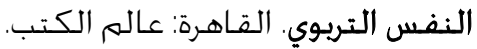

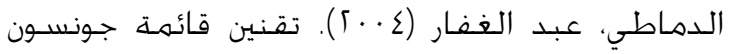

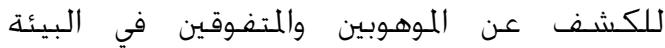

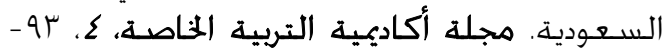
$.10 \mathrm{~V}$

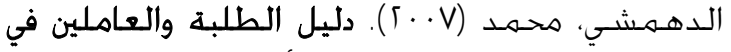

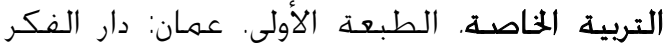
ناشـرون وهوزعون.

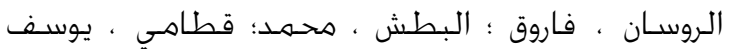

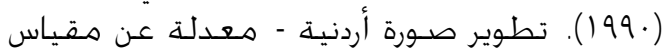

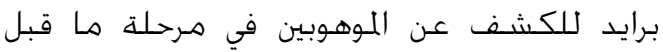

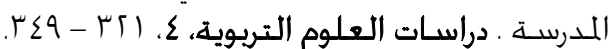
الروسـان، فاروق؛ الســرور، ناديا؛ الصـمادي، يحيى؛ العجلوني. خالد؛ أبو طالب، صـابر؛ باجس، إبراهيم (1991).

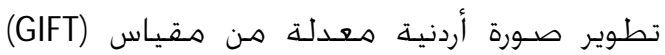

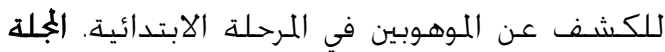

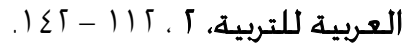

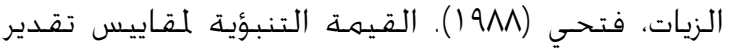

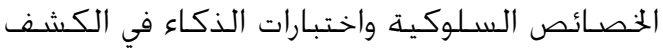

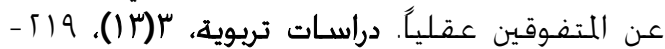
. 11

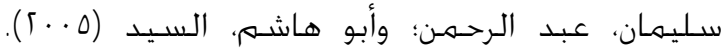

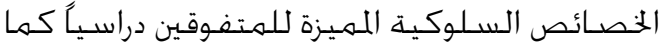

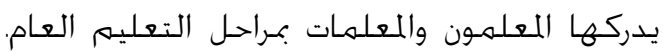

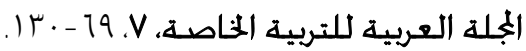

للتأكد هـ خصـائصها السيكوهترية، حيث أن تدريج

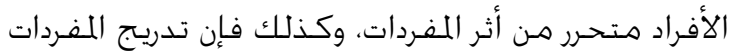
متحـرر هـن أثر الأفراد.

كما عكسـت نتائج هذه الـراسـة الصـورة الايجابية

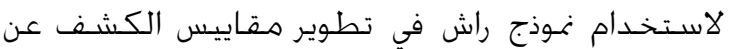

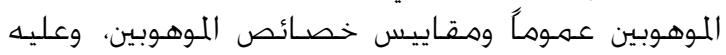

$$
\text { توصى الـراســة بالآتي: }
$$

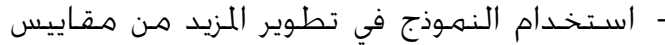

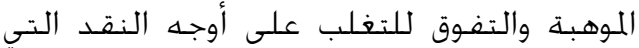
وجهت لتلك المقاييس.

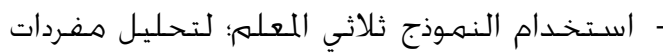

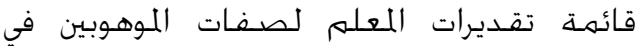

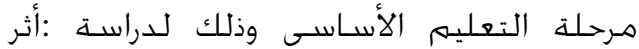
عاملي التخـمـين وقوة تمييز مفردات القـائمـة.

\section{المراجع}

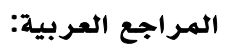

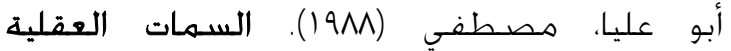

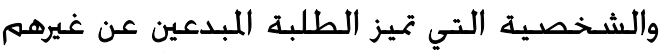

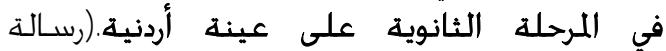

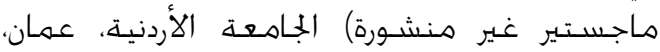

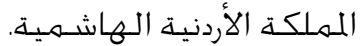

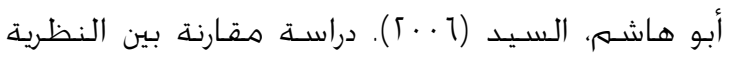

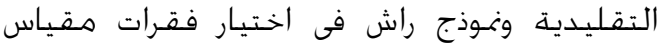

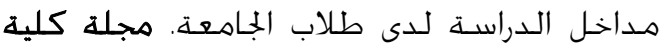

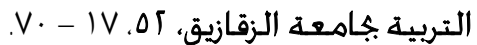

أيوب، حسـين (9MM). العلاقة بين بعض المتغيرات

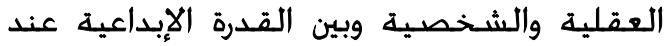

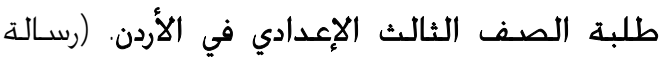

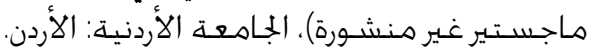
البطش، محمدد: الروســان، فاروق (1991). التحليل

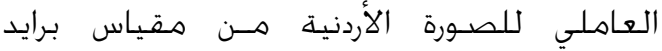

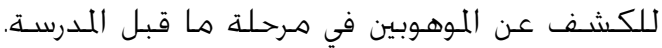

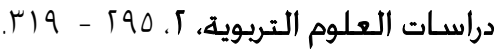
بن فاطمة، محهمد؛ معاوية، عبد اللّ؛ السـويسي، جناة

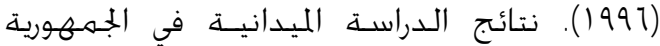

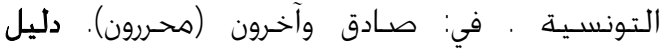
أسـاليب الكشف عن الأوني الموهوبين في التعليم

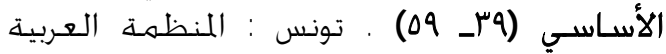
للثقافة والعلوم.

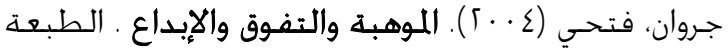

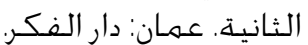

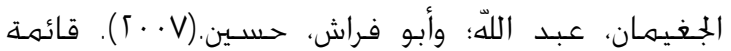

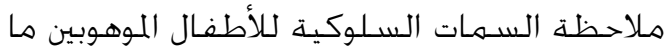


والتفكير الابتكاري. مجلة البحث في التربية وعلم

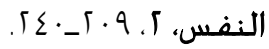

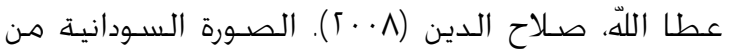

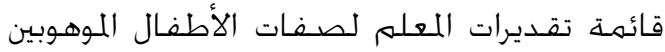

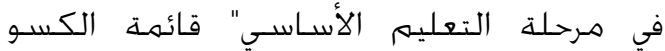

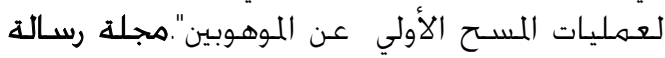

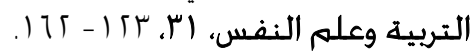
عطا اللّ، صسلاح الـين ( 1 . . ؟). الكشـف عن الموهوبين

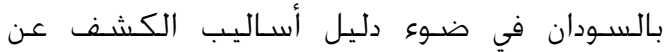

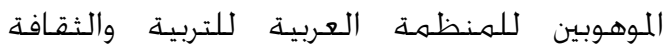

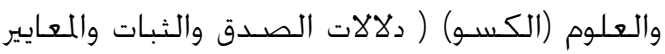

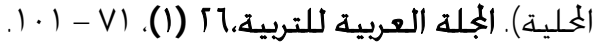

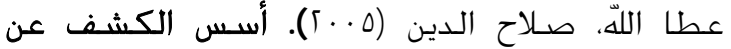

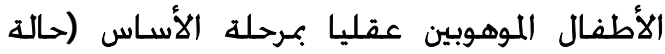

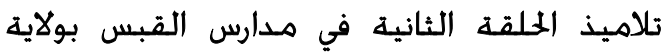

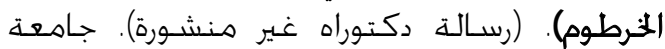
الخرطوم، الخـرطوم، جـهـورية السـودان.

عطيات، مظهر (1 · ؟). العلاقة بين بعض المتغيرات

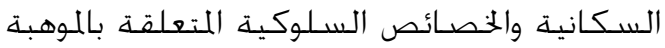
لدى أطفال ما قبل المدرسـة. مجلة جامعة الملك المكانك

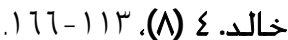
علام، صـاح الدين (910). خليل بيانات الاختبارات

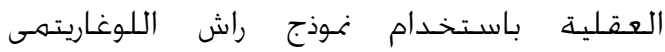

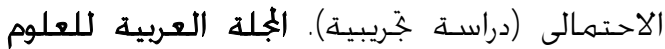

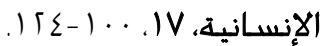
علام، صـلاح الدين (9MV) (1). دراسـة مـوازنة ناقدة لنماذج

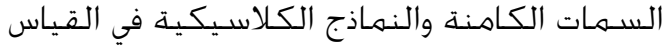
النفسي والتربوي. الجلة العربية للعلوم الإنسانية، $. \Sigma \Sigma-1 \Lambda .5 V$

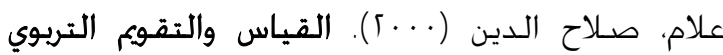
والنفسي - أساسياته وتطبيقاته وتوجهاته المعاصـرة. القـاهرة ، دار الفكر العربي.

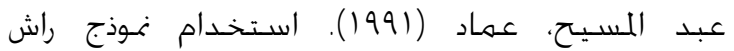

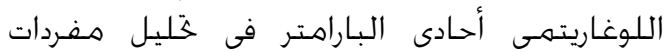

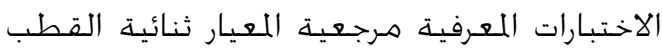

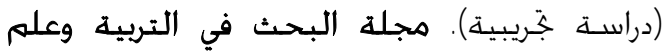

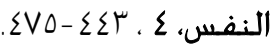

عودة، أحسمد (9951). مدى التوافق بين نموذج راش والمؤشـرات التقليدية في اختيـار فقـرات مقياس الجّاه

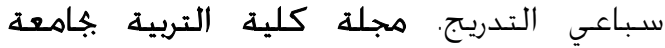

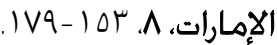

قبلان، بسام (990) (190ناء مقياس للكشف عن الطلبة

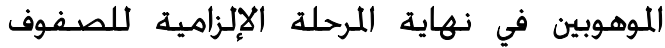

الشـافعي، رجب؛ محـمد، أحسمد (1991). مقياس "رم" (لأ

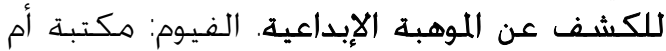
القـرى.

شـاكر، شـاكر (1990). علم نفس الإبداع. القـاهـرة : دار غريب للطباعة والنشـر.

شـــير، زينب ( ( • ؟).دليل المعلم والوالدين لتشخيص

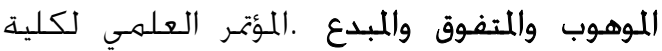
التربية بكامعة بنها (اكتشـاف ورعاية الموهوبين بين والمين

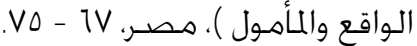

الشـمـري، فائزة (ه · ؟). تطوير صـورة كويتية لاختبار

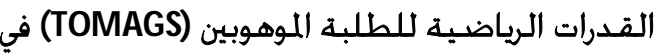
المرحلة الابتدائية. (رسـالة ماجســـير غير منشـورة). جامعة الخليتج العربي، المنامة، ملكة البهة البحـرين. صادق، آهال؛ السيد، عبد الخليهم: علام، صسلاح الدين

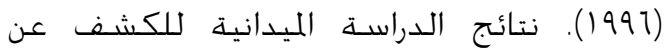
الموهوبين في جـهـورية مصـر العربية. في : صـادق.

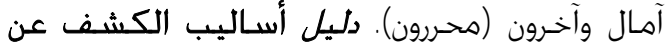

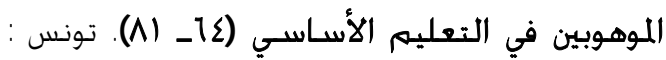

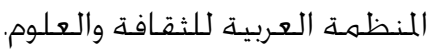

الصـوص، إيمان (1990). دراسـة مقارنة لسـمات الشخصية التي تميز الطلبة الموهوبين من العاديين

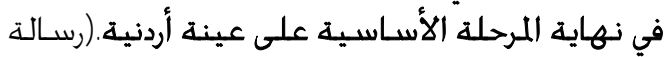

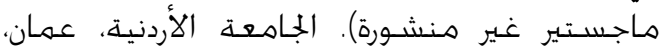

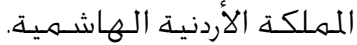

الطحان، محـمـد: أبو هلال، ماهـر (997 (). نتائج الـراســة

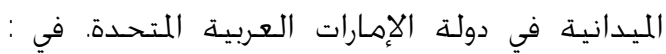

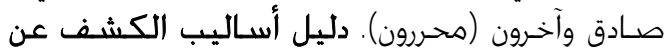

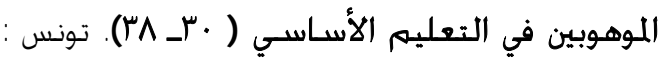

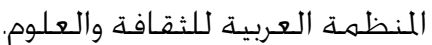

عبد الغفـار، عبد السـلام؛ و صـادق، آمال؛ والسـيد، عبد

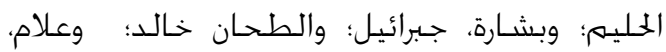

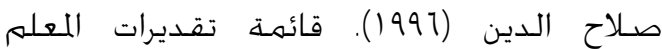

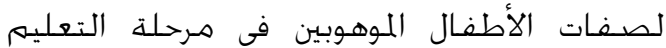
الأسـاسـي. في : صـادق وآخرون (محررون). دليل

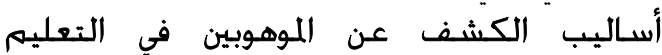
الأسساسي ( 90_9V). تونس : المنظمة العربية

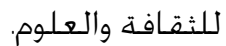

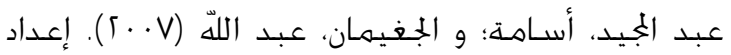

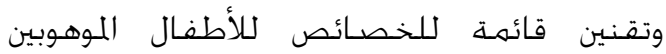

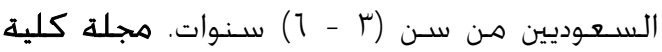

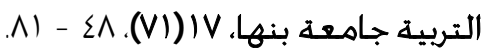

عبد الوارث، سـمية (997). الخصـائص السـلوكية

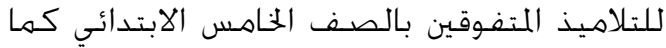

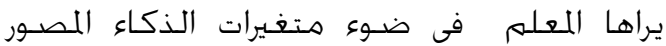




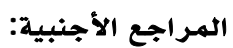

Argulewicz, E. (1981). Validity of the SRBCSS for hispanic and anglo gifted students. A paper presented at the Annual Meeting of the American Psychological Association. Los Angeles, Aug. 19-31. p. 21.

Argulewicz, E.(1985). Review of scales for rating the behavioral characteristics of superior students. In J. V. Mitchell Jr. (Ed.), The ninth mental measurements yearbook (Vol. 2, pp. 1311-1312). Lincoln, NE: Buros Institute of Mental Measurement of theUniversity of NebraskaLincoln.

Cummings, W. (1980). Cummings checklist of characteristics of gifted and talented children. Paper presented at the Annual International Convention of The Council for Exceptional Children (58th, Philadelphia, PA, April, 1980, Session W-94).

Davis, G., \& Rimm, S. (1974). Group inventory for finding interest (GIFFI) I and II: Instruments for Identifying creative potential in junior and senior high school. Journal of Creative Behavior ,16,50-57.

Davis, G., \& Rimm, S. (1980). GIFFI II: Group inventory for finding Interests. Watertown, WI: Educational Assessment Service.

Eby, J. (1983). Gifted behavior: Anon-elitist approach. Educational Leadership,41, 30-36.

Elliott, S., Argulewicz, E., \& Turco, T. (1986). Predictive validity of the scales for rating the behavioral characteristics of superior students for gifted children in three sociocultural groups. Journal of Experimental Education, 55, 27-32.

Elliott, S. N., Busse, R. T., \& Gresham, F. M. (1993). Behavior rating scales: Issues of use and development. School Psychology Review, 22, 313321.

Feldhusen, J. F., \& Heller, K. A . (1986). Introduction. In K. S. Heller \& J. F. Feldhusen (Eds.), Identifying and nurturing the gifted: $A n$ international perspective (pp. 19-31). Toronto, Canada: Hans Huber.

Feldhusen, J.,Hoover, S., \& Sayler, M. (1987). The Purdue academic rating scal es. Paper presented at the Annual Convention National Association for gifted Children. N ew Orleans, LA: USA.

Friedrichs, T. (1990). A teacher-based checklist for identifying giftedness in learning-disabled students: A validity study. Unpublished PhD Dissertation. University of Virginia: USA.

Grant, D. (1996). Screening for gifted: A factor analytic study of measures used for identifying giftedness. Unpublished PhD Dissertation . University of Southern Mississippi: USA.

$$
\begin{aligned}
& \text { (الثامن ، التاسـع ، العاشـر). (رسـالة ماجسـتير غير }
\end{aligned}
$$

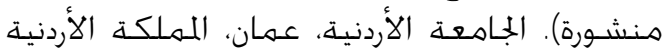

$$
\begin{aligned}
& \text { المهاشمية. } \\
& \text { كاظم، أمينة (1997). نماذج السـمات الكامنة في: أنور }
\end{aligned}
$$

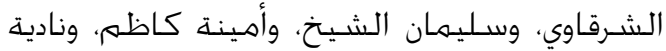

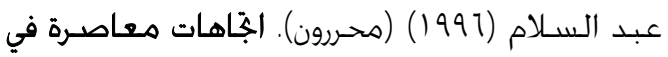

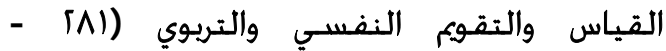

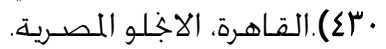

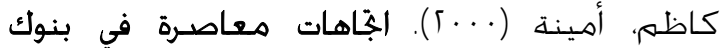

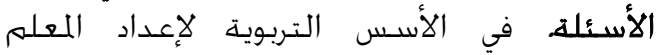

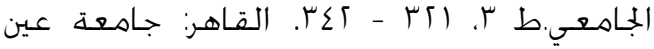

$$
\begin{aligned}
& \text { شـمس. } \\
& \text { كراجة، عبد القادر (1. (.). القياس والقويم في علم }
\end{aligned}
$$

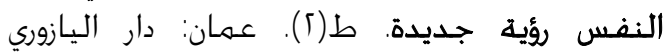

$$
\begin{aligned}
& \text { العلمية للنشـر والتوزيع. }
\end{aligned}
$$

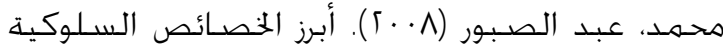

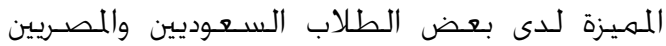

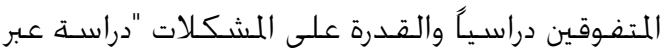

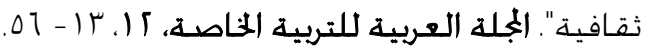

$$
\begin{aligned}
& \text { المطيري، حمد (0. (؟)). تطوير مقياس للتعرف على }
\end{aligned}
$$

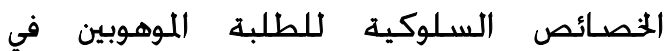

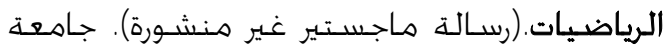

$$
\begin{aligned}
& \text { الخليـج العربي، المناهة، مـلكة البحـرين. } \\
& \text { منسي، محمـود (990 (). قائمة سـمات الأطفال المبتكرين }
\end{aligned}
$$

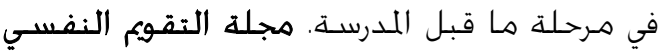

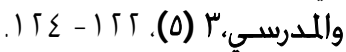


Hambleton, R. \& Swaminathan, H. ( 1989). Item response theory principles and applications. Boston: Kluwer Nijhoff Publishing.

Irby, B., \& Lara-Alecio, R. (1996). Attributes of hispanic gifted bilingual students as perceived by bilingual educators in Texas. SABE Journal, $11,120-143$.

Miller, R., \& Gentry, M. (2010). Developing talents among high-potential students from lowincome families in an out-of-school enrichment program. Journal of Advanced Academics, 21, 594-627.

Pfeiffer, S., Jarosewich, T. (2007). The gifted rating scales-school form: An analysis of the standardization sample based on age, gender, race, and diagnostic efficiency. Gifted Child Q uarterly, 51, (1), 39-50.

Pfeiffer, S., Petscher, Y., \& Jarosewich, T. (2007). The gifted rating scales-preschool/ kindergarten form: an analysis of the standardization sample Based on age, gender, and race. Roeper Review, 29 (3), 206-211.

Pfeiffer, S., Petscher, (2008). Identifying young gifted children using the gifted rating scales preschool/ kindergarten form. Gifted Child Q uarterly, 52 (1) ,19-29.

Pfeiffer, S., Petscher, Y., \& Kumtepe, A. (2008). The gifted rating scales-school form: A validation study based on age, gender, and race Roeper Review, 30 (2), 140-146.

Renzulli, J., Siegle, D., Reis, S., Gavin, M ., \& Reed, R. (2009). An investigation of the reliability and factor structure of four new scales for rating the behavioral characteristics of superior students. Journal of Advanced A cademics, 21(1), 84-108.

Renzulli, J., Smith, L. White,A., Callahan, C., \& Hartman, R. (1976). Scales for rating the behavioral characteristics of superior students. Mansfield Center, CT: Creative Learning Press.

Renzulli, J. S., Smith, L. H., White, A. J., Callahan, C. M., Hartman, R. K., \& Westberg, K. L. (2002). Scales for rating the behavior characteristics of superior students: Revised edition. Mansfield Center, CT: Creative Learning Press.

Rimm, S.(1976). GIFT: Group inventory for finding creative talent. Watertown, WI: Educational Assessment Service.

Rimm, S. (1983). Preschool and kindergarten interest descriptor (PRIDE) Manual for administration. Wisconsin: Educational Assessment Service Inc.

Rimm, S. (1988). AIM -TO: A chievement identification measure - teacher observation. Watertown, WI: Apple

Rudasill, K.; Callahan, C. (2008). Psychometric characteristics of the Harter self-perception profiles for adolescents and children for use with gifted populations. Gifted Child Quarterly, 52 (1), 70-86.

Rust, J. (1985). Review of scales for rating the behavioral characteristics of superior students. In J. V. Mitchell Jr. (Ed.), The ninth mental measurements yearbook (Vol. 2, pp. 312-313). Lincoln, NE: Buros Institute of Mental Measurement of the University of NebraskaLincoln.

Ryser, G.; \& McConnell, K. (2004). Scales for identifying gifted students. New York: Purfrock press Inc. Scales for rating the behavioral characteristics of superior students. Creative Learning Press, Inc. Mansfield Center, CT.

Scott, J., \& Marcia, G. (2010). Multigroup construct validity evidence of the HOPE scale: Instrumentation to identify low-income elementary students for gifted programs. Gifted Child Q uarterly, 54(4), 298-313.

Silverman, L. (2003). Characteristics of giftedness scale : A review of literature. Retrieved from: $\mathrm{Http//:www. \quad Gifted} \mathrm{development.}$ Com/characteristics.htm, 3 March 2010.

Vaughn-Neely, E. (1994). A study of the Kingore observation inventory as a screening procedure for the identification of highly able second graders. (Unpublished PhD Dissertation).Oregon State University, Portland, USA.

Waters, J. (1989). The Silverman/Waters checklist for identifying gifted elementary school-aged children: A validation study. (Unpublished PhD Dissertation).University of Denver, Denver, USA. 